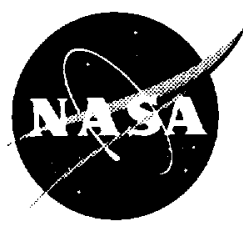

\title{
A Spherical Torus Nuclear Fusion Reactor Space Propulsion Vehicle Concept for Fast Interplanetary Travel
}

Craig H. Williams, Stanley K. Borowski,

Leonard A. Dudzinski, and Albert J. Juhasz

Lewis Research Center, Cleveland, Ohio

Prepared for the

34th Joint Propulsion Conference and Exhibit

cosponsored by the AIAA, ASME, SAE, and ASEE

Cleveland, Ohio, July 13-15, 1998

National Aeronautics and

Space Administration

Lewis Research Center 


\section{Acknowledgm ents}

The authors wish to thank many who have been very helpful in providing guidance and expertise: to Gerald Hale of Los Alamos National Lab (LANL) in the area of spin polarize d $D^{3} \mathrm{He}$ fuel, to Stanley Kaye of Princeton Plasma Physics Lab (PPPL) in the area of fusion confinement time scaling laws, to Joseph Warner of NASA Lewis Research Center (NASA LeRC) in the area of YBCO superconductor mate rial properties and prospects, to Ronald Moses of LANL in the area of Dshape magnet designs and forces, to Lawrence Green of Westinghouse Science and Technology Center and Daniel Driemeyer of Boeing Co. in the area of ITER divertor design drivers, to Peter Turchi and Hani Kamhawi of Ohio State University in the area of magnetic nozzle design and state conditions, to Larry Grisham of PPPL and Masaaki Kuriyama of the Japan Atomic Energy Research Institute in the area of negative ion neutral beam injectors, to Richard Kunath of NASA LeRC in the area of Ka band space communications, to Robert Cataldo of NASA LeRC in the areas of start-up NiH batteries and Mars human payload systems; to John Miller of JME, Inc. and Mary Ellen Roth of NASA LeRC in the area of high energy battery capacitors, to John Sankovic of NASA LeRC and R. Joseph Cassady of Primex Technologies Corp. in the area of high power hydrogen arcjets, to Mellissa McGuire of Analex Corp. in the area of reaction control, to Judith Watson of NASA Langley Research Center in the area of Space Station truss network, and to Mohamed Bourham of North Carolina State University and Stanley Milora of Oak Ridge National Lab in the area of fuel injectors.

Available from

NASA Center for Aerospace Information 7121 Standard Drive

Hanover, MD 21076

Price Code: A03
National Technical Information Service 5285 Port Royal Road Springfield, VA 22100

Price Code: A03 


\title{
A SPHERICAL TORUS NUCLEAR FUSION REACTOR SPACE PROPULSION VEHICLE CONCEPT FOR FAST INTERPLANETARY TRAVEL
}

\author{
Craig H. Williams', Stanley K. Borowski"*, \\ Leonard A. Dudzinski*** , Albert J. Juhasz ...* \\ NASA Lewis Research Center \\ Cleveland, $\mathrm{OH} 44135$ \\ (216) 977-7063, -7091, -7107, 433 - 6134
}

\begin{abstract}
A conceptual vehicle design enabling fast outer solar system travel was produced predicated on a small aspect ratio spherical torus nuclear fusion reactor. Initial requirements were for a human mission to Saturn with a $>5 \%$ payload mass fraction and a one way trip time of less than one year. Analysis revealed that the vehicle could deliver a $108 \mathrm{mt}$ crew habitat payload to Saturn rendezvous in 235 days, with an initial mass in low Earth orbit of 2,941 mt. Engineering conceptual design, analysis, and assessment was performed on all major systems including payload, central truss, nuclear reactor (including divertor and fuel injector), power conversion (including turbine, compressor, alternator, radiator, recuperator, and conditioning), magnetic nozzle, neutral beam injector, tankage, start/re-start reactor and battery, refrigeration, communications, reaction control, and in-space operations. Detailed assessment was done on reactor operations, including plasma characteristics, power balance, power utilization, and component design.
\end{abstract}

\section{INTRODUCTION}

The impetus for this effort was three fold. First: in order to guide the long range NASA goal of human expansion throughout the solar system, a rational approach for long term advanced research and development must be clearly articulated. Second: currently funded nuclear fusion space propulsion research must continually be shown to be on the critical path for enabling order-of-magnitude improvements in future space transportation capability. Third: a conceptual vehicle design incorporating the proposed design philosophies and related results of a recent series of NASA Lewis Research Center (LeRC) papers was the logical next step in the process ${ }^{1,2,3}$.
The findings of these earlier papers emphasized that for piloted, outer solar system missions expected within the $21^{\text {st }}$ century, adequate payload mass fraction (5\% to $15 \%)$ and multi-month trip times would require specific impulses $\left(\mathrm{I}_{\mathrm{sp}}\right)$ and specific powers $(\alpha)$ of 20,000 to $50,000 \mathrm{lb}_{\mathrm{f}} \mathrm{sec} / \mathrm{b}_{\mathrm{m}}$ and 5 to 50 $\mathrm{kW} / \mathrm{kg}$ respectively ${ }^{1,3}$. Although contestable, it is the judgment of the authors and many in the field that only a single space propulsion technology exists at this time that can reasonably be expected to offer this capability: nuclear fusion, either magnetic or inertial confinement.

Nuclear fusion reactors can be broadly classified into at least three groups: closed magnetic (such as tokamaks, small aspect ratio toroids, spheromaks, field reversed, etc.), open magnetic (mirrors), and inertial concepts. Based in part on the results of previous studies ${ }^{1,4}$ of the attributes and shortcomings of these reactor groups towards space propulsion, a closed magnetic system was chosen for this design concept. The high power density achievable in closed systems, improved confinement, density and temperature profile peaking, and spin polarization of the fuel, provided a distinct advantage in their application towards space propulsion. Further, while the large aspect ratio tokamaks have been the predominant focus of the fusion research community for many years, their great size and mass render them unappealing for space propulsion, where light weight is paramount. At the other end of the closed reactor spectrum, spheromak and field reversed concepts offer tremendous hope for compact, light weight propulsionoriented systems. However, the dearth of experimental data on their operation and indeed their engineering feasibility render serious engineering assessment difficult. As a result, a compromise was struck between existing, extrapolatable experimental databases from tokamaks and the largely conceptual compact toroids. The small aspect ratio spherical torus, a concept

- M.E., P.E./Aerospace Engineering, Senior Member AIAA

"Ph.D./Nuclear Engineering, Senior Member AlAA

** B.S./Aeronautical and Astronautical Engineering, Member AIAA

.... M.S.E./Mechanical and Aeronautical Engineering, Senior Member AIAA 
midway between these two groups, was thus chosen to serve as the basis for the vehicle concept.

Consistent with the "top-down" requirementsdriven approach documented elsewhere', this vehicle design concept was initiated by first establishing a simple set of mission requirements, then producing a consistent engineering design that satisfied those requirements. This meant that current state of the art systems, along with experimental results, were used as the basis to extrapolate to what could be technologically available to a human presence solar system-wide of the not too distant future -- 30 years from now. All system engineering analysis was performed using existing computer programs, open literature engineering sources, and basic engineering calculations. The preponderance of the nuclear fusion engineering data was obtained from Department of Energy terrestrial power and scientific research programs, while much of the propulsion system engineering data was derived from NASA expendable launch vehicle and conceptual nuclear thermal rocket (NTR) design studies. Only limited NASA fusion space propulsion system data exists beyond what was accomplished since the termination of the 20 year long fusion propulsion program at NASA LeRC in 1978.

\section{MISSION REQUIREMENTS}

The top-down, mission requirements-driven design process began with specifying the desired mission, trip time, and payload mass fraction. These requirements were then used to define operation parameters: mission distance, specific power, specific impulse, and nozzle jet efficiency. These operation parameters are directly related to four primary system characteristics: structure mass, power out of reactor, jet power, and thrust. The primary system characteristics were the focus of the engineering design effort, where iteration was conducted until the mission requirements were satisfied.

The vehicle concept was to be able to perform a rendezvous (one way) mission to the Saturn, piloted by a crew of six, with a $>5 \%$ payload mass fraction, and a trip time of less than one year. Performance assessment of the same vehicle was also to be made for a similar mission to Jupiter. Also investigated was the propellant required to arrive at the specific destinations at Saturn and Jupiter: their major moons Titan and Callisto respectively. The Titan mission was selected due to its demanding performance requirement, scientifically interesting possibility of life on its surface requiring human presence for investigation, its dominant size among the Saturnian satellites, its expected abundance of hydrogen for propulsion application, and the abundance of fusion fuels $D_{2}$ and ${ }^{3} \mathrm{He}$ in the planet's atmosphere. The Callisto mission was selected for similar reasons, but also to represent a less performance demanding mission for comparison purposes (as well as the Galilean satellite farthest outside of the Jovian gravity well). The mission distance was predicated on optimal planetary orientation, though performance requirements for more demanding planetary positions were also calculated.

The exploratory and scientific nature of the mission drove the requirement for human presence. Based primarily on existing humans to Mars mission studies, a reasonable crew size of six was chosen. The crew habitat payload design and its requirements, being outside the scope of this study, were largely adopted and scaled-up from current Mars mission spacecraft designs. Although an ample $(>5 \%)$ payload mass fraction was initially set as a requirement, even a generousìy scaled-up Mars mission payload mass was found to result in a lower payload mass fraction due to the excessive total mass of the entire vehicle concept. Therefore, an explicit payload mass fraction was replaced with a specific payload mass value.

The piloted nature of the mission also drove the requirement for relatively fast trip times. The one year maximum was somewhat arbitrary, but was represent ative of long duration human experience in low Earth orbit and consistent with some current Mars mission studies. The intent was to force the vehicle concept to perform multi-month (vs. multi-year) missions, despite interplanetary distances that dwarf the more commonly thought of Earth to Mars transfers.

\section{VEHICLE OVERVIEW}

Figures 1 through 4 illustrate the overall layout $o^{-}$the vehicle concept. The cylindrical crew habitat pilyload was forward of the propulsion system. It was linked to the central truss through an adapter which also attached to the avionics suite and truss booms supporting the communication antennas. The forward sentral truss supported the four square panel heat reje sting radiators. Along the outside of the mid central tr uss were six slush hydrogen propellant tanks. Within th e mid central truss was the $D^{3} \mathrm{He}$ fuel tank and refrigeration system for all propellant/fuel tankage. Throughwut the central truss were also various data, power, coolant, and propellant lines. Outside of the aft central truss were two Brayton power conversion systems, including recuperators. Within the aft central truss were the power management and distribution system, the neutral beam injector, the refrigeration system for all the superconducting magnetic coils, and the start/re-start reactor and battery bank. Aft of the 
Figure 1: Vehicle Concept Overview (Aft View)

Table 1: Mass Property Summary reactor and the magnetic nozzle. The overall vehicle length was $260 \mathrm{~m}$. The greatest deployed system dimension was $150 \mathrm{~m}$ of the square heat rejection (radiator) system and its connecting central truss. However the maximum stowed diameter for any individual system was limited to $10 \mathrm{~m}$ so as to fit within a reasonable payload fairing, facilitating launch and onorbit assembly. The fully tanked initial mass in low Earth orbit (IMLEO) was 2,941 mt.

The design philosophy followed was to locate as much of the vehicle mass (power conversion, reactor, and propellant) as close to and forward of the thrust vector as possible to facilitate steering control authority. Although shielded and emitting limited neutron radiation, the reactor was kept as far aft of the crew payload as possible. The modular vehicle layout, system packaging, launch operations, and in-space assembly sequence was expected to be conducted in a way that maximized docking maneuvers rather than labor intensive Extra Vehicular Activity (EVA). Since the vehicle was designed for interplanetary cruise, only two docking ports, located forward and side, were provided to accommodate surface-based landing craft.

\begin{tabular}{llrr}
\hline Payload & & & 108 \\
D & & 45 \\
Hydrogen fuel & propellant & \multicolumn{2}{r}{1,292} \\
& Main impulse & 1,220 & \\
& Reaction control & 21 & \\
& Flight performance reserve & 12 & \\
& Residuals/losses & 39 & \\
Structure & & \multicolumn{2}{c}{1,496} \\
& Central truss & 15 & \\
& Reactor & 436 & \\
& Power conversion & 381 & \\
& Refrigeration & 54 & \\
& Magnetic nozzle, divertor & 8 & \\
& Neutral beam injector & 65 & \\
& Start/restart reactor, battery & 11 & \\
& Reaction control & 48 & \\
& Avionics, communication & 2 & \\
& Cryo-tankage & 131 & \\
Weight growth contingency & 345 & \\
\hline IMLEO(mt) & & 2,941 \\
\hline
\end{tabular}



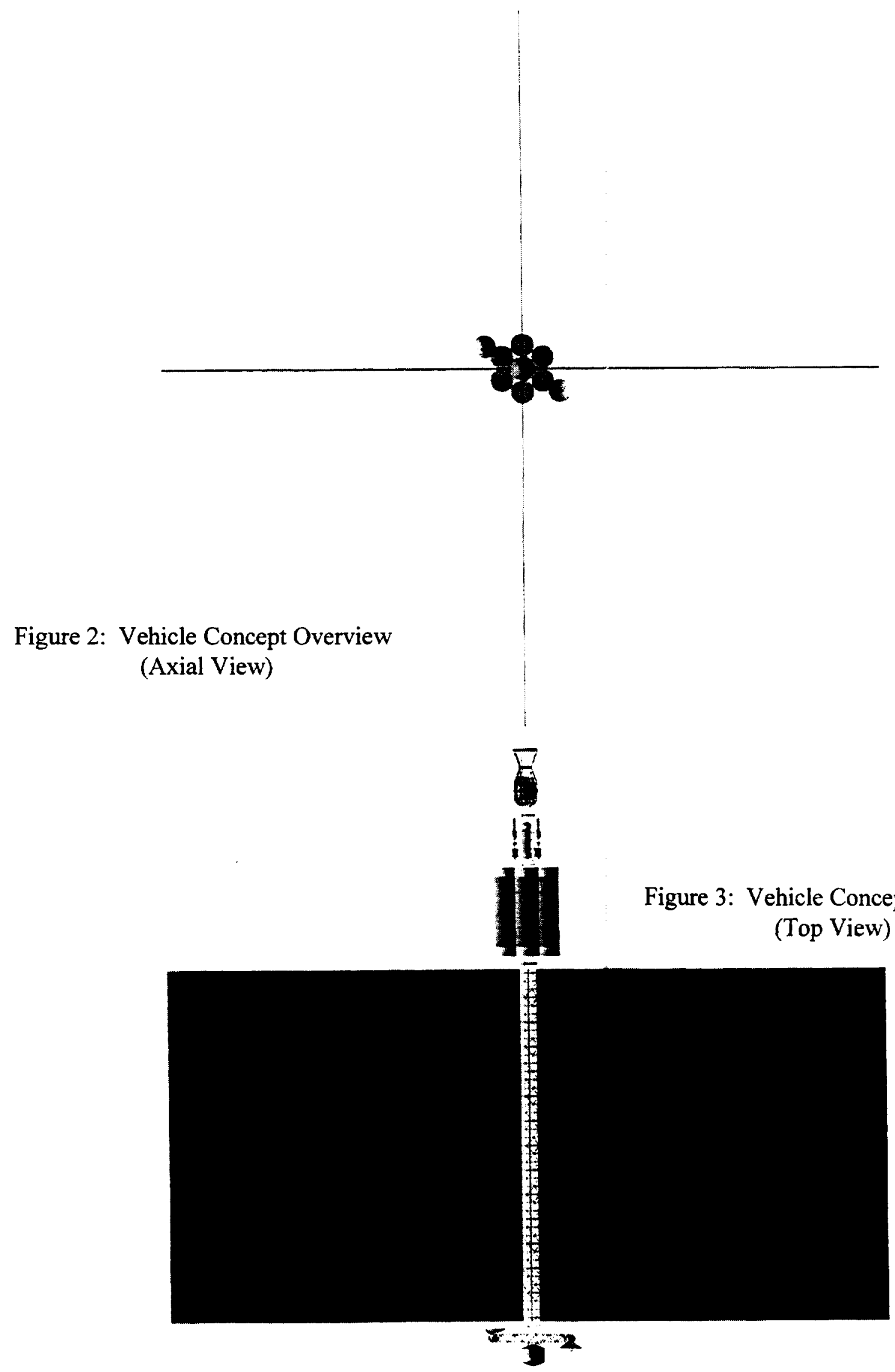
Table 1 illustrates the mass property summary for the fully loaded stack. The "payload mass" was 108 $\mathrm{mt}$ and consisted of useful payload only (crew, habitat, consumables, scientific instruments, etc.) The "fuel" was $45 \mathrm{mt}$ and was solely $\mathrm{D}^{3} \mathrm{He}$ used to fuel the reactor. The "propellant mass" was 1,292 $\mathrm{mt}$ and was slush hydrogen for momentum transfer, reaction control, reserves, and losses. It does not include system or tankage mass. (For the purposes of calculating the velocity ratio to be defined in the following section, the propellant mass $\left(M_{p}\right)$ was restricted to only that for the main impulse. The balance of slush hydrogen (reserves, etc.) was book-kept with the structure mass.) The total structure mass $\left(\mathrm{M}_{\mathrm{s}}\right)$ was $1,496 \mathrm{mt}$ and referred to all mass required to operate the propulsion system.

\section{MISSION ANALYSIS}

Fusion propulsion systems are expected to operate at high enough $I_{s p}$ and $\alpha$ to produce accelerations greater than the local acceleration due to solar gravity at Earth's orbit $(0.6$ milli-g; where 1 milli$\left.\mathrm{g}=32.173910^{-3} \mathrm{ft} / \mathrm{sec}^{2}\right){ }^{\prime}$. The normally thought of conics of minimum energy trajectories followed by today's chemical systems degenerate into nearly straight line, radial transfers at these high acceleration levels with continuous thrust. A "field-free space" approximation can be invoked to greatly simplify the usually complex orbital mechanics. Gravity losses and optimum steering concerns can be neglected without introducing too much error, obviating the need for computationally intensive, numerically integrated solutions to support preliminary analysis. In addition, a "launch at anytime" approach to mission design is a luxury that can usually be assumed for fusion systems so long as the thrust to weight is great enough compared to the local acceleration due to solar gravity. As will be shown, despite an initial thrust to weight of only 0.887 milli-g , the vehicle concept's trajectory was reasonably close to that of a radial transfer.

At the initiation of and throughout the design effort, a simple high $\mathrm{I}_{\mathrm{sp}}$ /high thrust algorithm ${ }^{3}$ for radial transfers was used to initiate and guide the design process and monitor convergence towards study requirements. This analytic, closed-form solution previously published by the co-author was used for the initial trip time and performance analysis. The governing relations are based on the classic rocket equation and auxiliary relations for high (constant) thrust, constant mass flow rate (variable acceleration) travel through field-free space.

At the conclusion of the design effort, the high

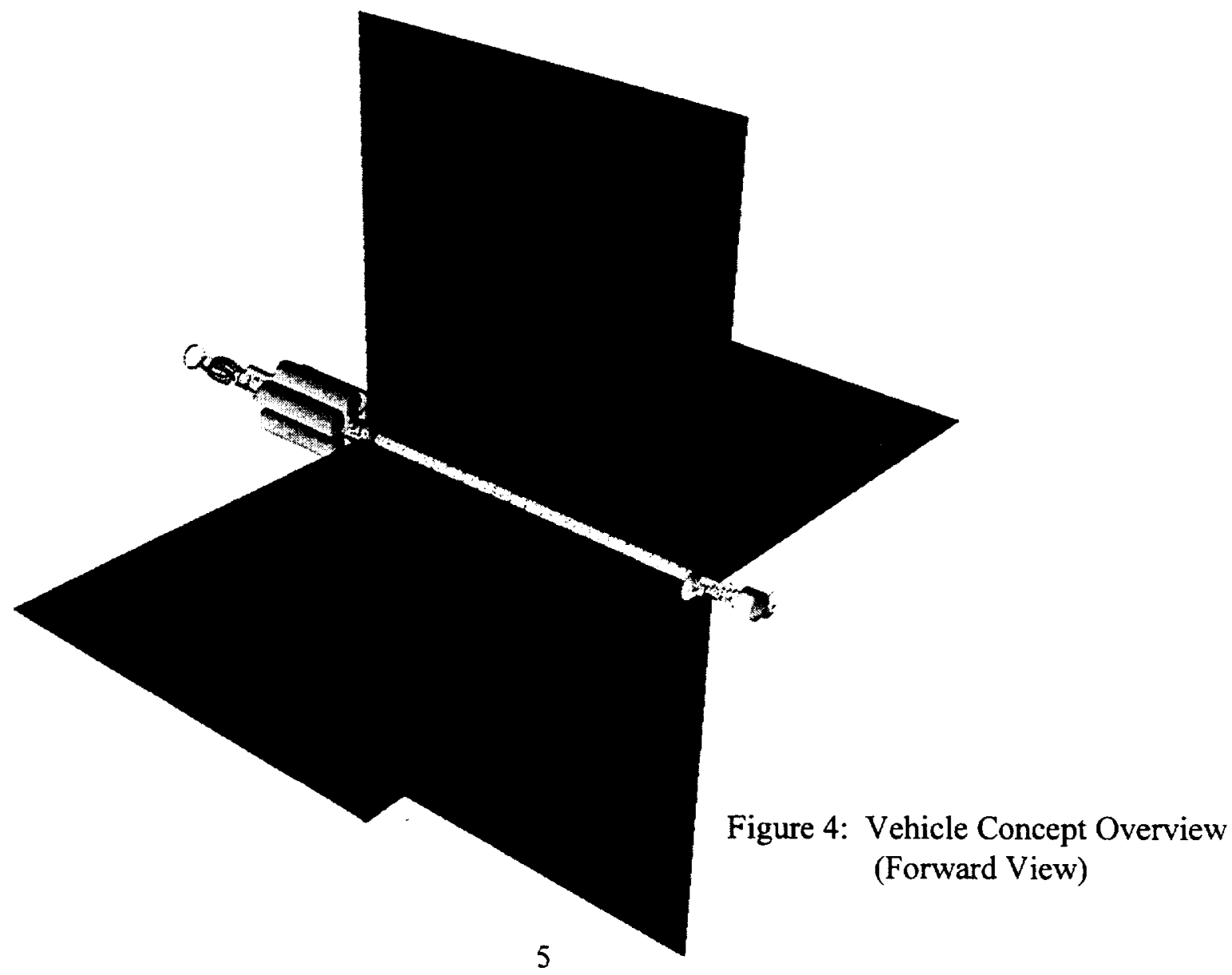


fidelity, variational calculus-based trajectory optimization program VARITOP was used to verify vehicle performance and overall mission design. VARITOP is a two body, heliocentric transfer computer program for modeling low thrust space propulsion systems ${ }^{s}$. It is well known throughout the preliminary design community for its good accuracy in solving the two point boundary value problem and integrating state variables. Good agreement was found between the two trajectory design computer programs. Figure 5 illustrates a heliocentric view of the integrated VARITOP trajectory from Earth to Saturn.

A few definitions of operational characteristics and system parameters are as follows. Specific power $(\alpha)$ is calculated differently throughout the space propulsion community. Here we will use the common, though perhaps not universal, definition of the ratio of power out from the reactor system $\left(\mathrm{P}_{\mathrm{out}}\right)$ (and sent into the thrust generating device) divided by $M_{s}$ (including reaction control propellant, reserves, and residuals) (equation (1)). $\alpha$ for the concept was $3.92 \mathrm{~kW} / \mathrm{kg}$.

$$
\alpha \equiv \frac{P_{a u t}}{M_{s}}
$$

The nozzle jet efficiency $\left(\eta_{j}\right)$ is the effectiveness of converting transport power out of the reactor into directed jet power in the thrust exhaust as defined in equation (2). The $\eta_{J}$ for this class of systems remains largely conjecture, consequently a value of 0.8 (i.e. $80 \%$ ) was assumed based on known low power electric propulsion systems and analytically derived minimum fusion system efficiencies ${ }^{6,7}$.

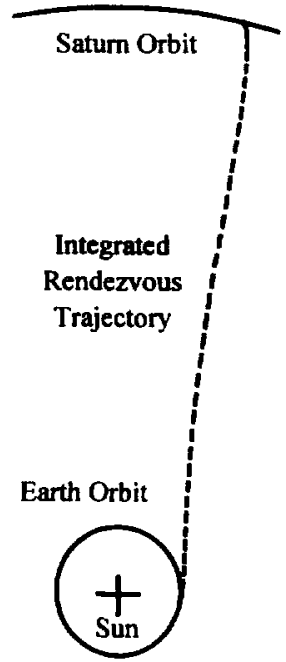

Figure 5: Integrated Earth - Saturn Trajectory (Heliocentric View)
Tatle 2: Performance Analysis Results

\begin{tabular}{|c|c|c|}
\hline Destination & Saturn & Jupiter \\
\hline Travel distance (AU) & 9.1 & \\
\hline$\alpha(\mathrm{kW} / \mathrm{kg})$ & 3.92 & same \\
\hline$I_{s p}\left(l b_{f} \sec / l b_{m}\right)$ & 40,485 & 32,590 \\
\hline Payload mass (mt) & 108 & same \\
\hline IMLEO (mt) & 2,941 & 2,925 \\
\hline Rendezvous trip time (days) & 235 & 150 \\
\hline Jet powe $(M W)$ & 4,916 & same \\
\hline$\eta_{J}$ & 0.8 & same \\
\hline Thrust $\left(b_{\mathfrak{f}}\right)$ & 5,567 & 6,916 \\
\hline $\mathrm{M}_{\mathrm{p}} \operatorname{dot}(\mathrm{kg} / \mathrm{sec})$ & 0.062 & 0.096 \\
\hline $\mathrm{c} / \mathrm{V}_{\mathrm{c}}$ & 1.113 & 1.120 \\
\hline Thrust/mass (initial) (milli-g) & 0.887 & 0.928 \\
\hline
\end{tabular}

Using these definitions and constant total mass flow rate ( $\dot{m}_{\text {total }}$, the total flow rate of propellant and reactor fuel and $\mathrm{g}_{\mathrm{c}}=32.1739 \mathrm{lb} \mathrm{ft} /\left(\mathrm{lb}_{\mathrm{f}} \mathrm{sec}^{2}\right)$ ), the jet thrust power $\left(\boldsymbol{P}_{\mathrm{jet}}\right)$, thrust $(\mathrm{F}), \mathbf{I}_{\mathrm{sp}}$, and exhaust velocity (c) can all be solved for using the familiar equation (3):

$P_{\text {jet }}=\eta_{J} P_{\text {out }}=\frac{F g_{c} I_{s p}}{2}=\frac{F^{2} g_{c}}{2 \dot{m}_{\text {total }}}=\frac{\dot{m}_{\text {total }} c^{2}}{2 g_{c}}$

Table 2 contains the overall performance analysis results for the design concept. All vehicle mass properties, including propellant loading, $P_{\text {out }}, \eta_{1}$ (and thus $\left.P_{j e s}\right), \alpha$, and payload mass were fixed for both missions. The $\mathrm{I}_{\mathrm{sp}}, \mathrm{F}$, and $\dot{m}_{\text {total }}$ were solved to satisfy the mission. For the Saturn rendezvous mission, the thrust v'as $5,567 \mathrm{lb}_{\mathrm{f}}, \mathrm{I}_{\mathrm{sp}}$ of $40,485 \mathrm{lb}_{\mathrm{f}} \mathrm{sec} / \mathrm{lb}_{\mathrm{m}}$, and a $\dot{m}_{\text {total }}$ (f $0.062 \mathrm{~kg} / \mathrm{sec}$. For the Jupiter rendezvous mission the thrust was $6,916 \mathrm{lb}_{\mathrm{f}}, \mathrm{I}_{\mathrm{sp}}$ of $32,590 \mathrm{lb}_{\mathrm{f}}$ $\mathrm{sec} / \mathrm{lb}_{\mathrm{m}}$, and a $\dot{m}_{\text {total }}$ of $0.096 \mathrm{~kg} / \mathrm{sec}$. Rendezvous missions were integrated for the optimal departure dates fom Earth to Saturn/Jupiter using the same payload module of $108 \mathrm{mt}$. The 235 day ( 8 month) trip time to Saturn and 150 day ( 5 month) trip time to Jupiter are rapid compared to trip times of represe itative alternate concepts. A similar rendezvous missior trip time to Saturn using chemical or even nuclear thermal propulsion would be measured in years. An analytic approximation was made of the trip time for opposition orientation of the planets. The Earth-Saturn trip time was lengthened by only $15 \%$, illustraing the relative insensitivity to launch date due to the high thrust to weight capability. These results 
demonstrate that the vehicle concept could accomplish fast interplanetary trip times with significant payloads over broad launch opportunities. Further improvements in payload capability and trip time could be achieved if fusion technology advances to permit the use of optimal propellant and payload mass fractions.

The vehicle concept was designed to carry a sub-optimal payload mass with a non-optimal propellant loading, contrary to earlier study recommendations'. Nuclear fusion technology offers perhaps the greatest useful power out for high thrust/high $I_{s p}$ propulsion technology appropriate for interplanetary mission requirements. But its $\alpha$ for credible propulsion designs, coupled with $M_{s}$ in excess of $1,000 \mathrm{mt}$, is still too low for optimum payload and propellant mass allocations below several 1,000's mt. Simply put, projected fusion propulsion systems are still too massive despite the large jet power they produce. The ratio of exhaust to characteristic velocities $\left(V_{c}\right)\left(c / V_{c}\right.$, a convenient measure of optimal propellant loading with respect to $M_{s}$ ) vs. payload mass fractions for representative mission difficulty factors $\left(\Delta V / V_{c}\right)$ (where velocity increment $(\Delta V)$ ) are illustrated in figure 6, with the design concept also plotted. It shows that the design concept was not optimal $\left(\Delta V / V_{c}=\right.$ $0.626)$ and considerably removed from the maximum potential payload fraction (or similarly, propellant loading). An optimal propellant loading for the same propulsion system mass and $\Delta V / V_{c}$ would have been $4,100 \mathrm{mt}$, representing 20 propellant tanks (and equivalently propeliant tank launches) as opposed to the 6 baselined. Less than 6 propellant tanks would have driven the $c / V_{c}$ to even greater values, lengthening

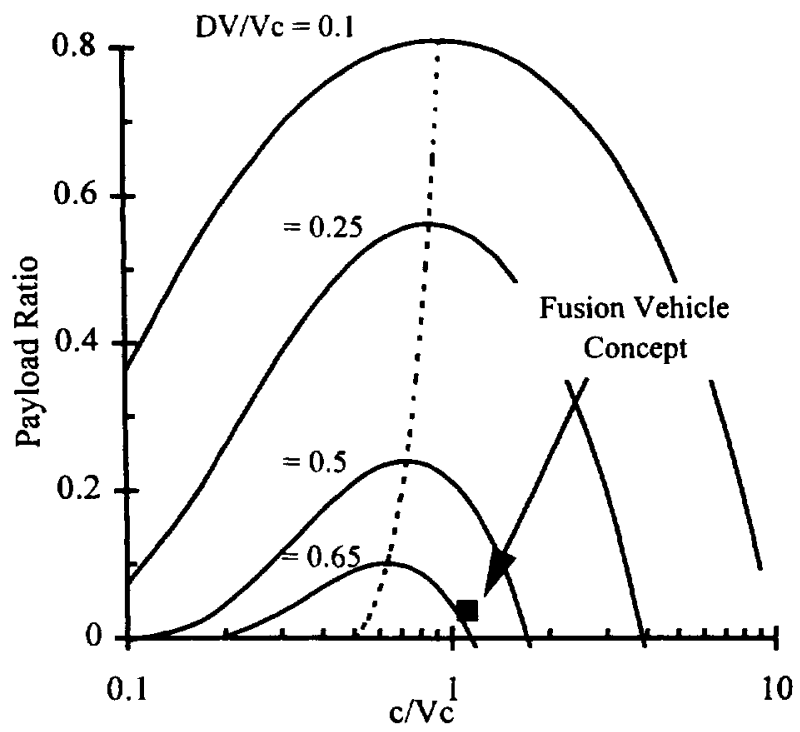

Figure 6: Payload Mass Fractions vs. $\mathrm{c} / \mathrm{V}_{\mathrm{c}}$ trip times and driving the concept further from the optimal value of $\mathrm{c} / \mathrm{V}_{\mathrm{c}}=0.65$. Thus a 6 propellant tank configuration was chosen, yielding a non-optimal, though otherwise reasonable $c / V_{c}=1.11$.

\section{SPACE OPERATIONS}

Space operations issues pertain to IMLEOdriven Earth to orbit (ETO) launch requirements, assembly and departure/arrival park orbit basing, and rendezvous vs. round trip propellant loading modus operandi. These issues are interrelated and have a profound influence on total system viability.

The large $M_{s}$-driven IMLEO represented a fundamental obstacle to viable space operations. The vehicle concept's IMLEO was $\sim 30$ times what could be delivered to LEO by a launch system in the Space Shuttle-class (that is: $80 \mathrm{mt}$ (orbiter) $+22 \mathrm{mt}$ (payload) $=102 \mathrm{mt}$ to a $140 \mathrm{nmi}$ circular orbit inclined 28.5 degrees) ${ }^{8}$. This could be accomplished with the socalled "Shuttle-C" booster, a long proposed derivative of the existing system that would operate without the Shuttle orbiter. More likely, a new heavy lift launch vehicle (HLLV) would be required. Sized for the greatest single payload masses and volumes, the HLLV payload capability could be as great as $\mathbf{2 5 0} \mathrm{mt}$ to LEO. This throw-weight capability of almost 2 "equivalent three stage Saturn V's", was at the upper range of past design studies ${ }^{9}$ and would be required to deliver a single, fully loaded propellant tank to LEO. Even this class of launch vehicle would require a dozen HLLV's to launch the initial configuration (and a half dozen for subsequent propellant re-loading, on a mass basis only).

Even with a new HLLV, a serious viability issue would still exist regarding launch availability. The ability to launch up to a dozen HLLV's within a reasonable time period will remain a dubious proposition for the foreseeable future. And launch costs associated with that many HLLV's would represent a significant percentage of total mission costs. Thus, dramatic reductions in launch processing and increases in robustness in launch availability would be mandatory for such a launch campaign to be viable.

On-orbit assembly would be a necessity for this concept. Individual systems would be configured to maximize simpler rendezvous and docking techniques as opposed to telerobotics or labor intensive EVA. A significant amount of on-orbit operations will still nonetheless require human presence despite the significant complexity, cost, and human factors issues surrounding EVA. Major systems (payload, central truss, heat rejection, etc.) would be assembled/deployed in orbit sequentially, facilitating these operations rather than attempting to minimize gross vehicle size by 
maximizing final vehicle configuration packing density. Assembly orbit altitude will most likely be no higher than $260 \mathrm{nmi}$ due to human radiation exposure limits and HLLV performance limits, while minimum orbits much below $140 \mathrm{nmi}$ are unlikely due to long term atmospheric drag and monatomic oxygen effects. Expensive launch and operations, coupled with the already high cost fusion system technology, will mandate the design requirement for long life and extended re-use. If fusion concepts actually prove to be as massive as the current design suggests, limits on onorbit assembly, launch availability, and maximum practical HLLV performance and volume capability could prove development-lethal for fusion propulsion.

The vehicle concept was designed for interplanetary cruise, thus a high altitude, sub-parabolic orbit space basing would obviate the need for multiweek spiral escapes and captures at its origin and destination. This also lessened the operation limits that would otherwise be imposed on a vehicle that is a source of high energy radiation and neutrons, particularly near populated areas such as a space station. These departure/arrival orbits could be low lunar, lunar-altitude, or Lagrange orbits at the Earth, and at very high minor moon or sub-parabolic orbit at the major planet destinations. This would require autonomous propulsion systems, possibly solar electric, to transport and change-out propellant tanks at departure and arrival points following their launch on HLLV's (Earth) and yet to be determined launch vehicles at the outer planets. Crew transport between Earth/Titan/Callisto and the vehicle concept could be performed by small, high thrust propulsion vehicles designed for fast orbit transfer.

Rendezvous missions were selected as the modus operandi due to their enabling of dramatic reductions in propellant requirements, vehicle size, and improved performance ${ }^{3}$ compared to carrying sufficient propellant for round trip missions. The implied requirement of a planetary refueling capability is of great concern, but is consistent with a solar systemclass transportation system regularly journeying to and between large outer planets with atmospheres and moons rich in $\mathrm{H}_{2}, \mathrm{D}_{2}$, and ${ }^{3} \mathrm{He}$. Sources of available propellant near high departure/arrival orbits, such as water ice at the lunar poles, minor moons, outermost major moons, and even asteroids, would greatly facilitate refueling without entering into deep gravity wells, provided the facilities could be established and maintained at a sufficiently low cost. It is reasonable to assume that in the time frame of fusion propulsion systems, other technologies and infrastructure (such as semi-robotic mining encampments) would be available. $D^{3} \mathrm{He}$ fuel would be acquired by either collecting solar wind deposited or scavenging in situ major planetary atmospheric deposits (if cost effective), which would alleviate the ${ }^{3} \mathrm{He}$ supply issue. Processing $40 \mathrm{~km}^{2}$ of lunar regolith to a depth of $2 \mathrm{~m}$, for example, could yield 1 and $6,100 \mathrm{mt}$ of ${ }^{3} \mathrm{He}$ and $\mathrm{H}_{2}$ respectively ${ }^{10}$. If these high orbit basing facilities were not available (or prove too expensive) and all propellant had to be supplied from terrestrial sources for entire round trip missions, then operations could become overwhelming.

Although the mission analysis assumed starting and stopping outside the effective gravity wells of the origin and destination planets, the sub-hyperbolic $\Delta \mathrm{V}$ propellant requirements were calculated for the Saturn mission. For the vehicle concept to go out of and in to deep gravity wells, the additional propellant mass would be as much as $51 \mathrm{mt}$ for Earth escape and $22 \mathrm{mt}$ for Saturn orbit capture at Titan. The corresponding spiral out and spiral in trip times were estimated to be 10 days and 5 days respectively. Thus escape and capture maneuvers would have a minor, though not insignificant, effect on propellant loading and trip time.

\section{PAYLOAD SYSTEM}

There have been several thorough engineering studies of human spacecraft for Mars missions during the last few years. The Transit Habitat from the recent NASA Human Exploration of Mars study" ${ }^{1}$ represents a compilation of much of these engineering design analyses. Consequently, it was decided to largely adopt the Transit Habitat for the design of the crew habitat payload system. The Transit Habitat's gross dimensions and mass were then scaled-up to accommodate the longer trip times for outer solar system missions.

The original Transit Habitat for Mars was a $7.5 \mathrm{~m}$ diameter, $7.5 \mathrm{~m}$ total height cylinder, two level structure able to accommodate a crew of six for a 180 day trip to Mars followed by provisions for up to a 600 day surface excursion ". The total habitable volume was $\sim 265 \mathrm{~m}^{3}$. This design was scaled-up for an up to one year mission by matching the diameter to that of the other $\mathrm{mi}$ jor systems $(10 \mathrm{~m})$ and increasing the total internal height proportionately to $6.75 \mathrm{~m}(9.2 \mathrm{~m}$ external), where the total habitable volume then equaled twice the original Habitat's $\left(530 \mathrm{~m}^{3}\right)$. This enabled twice the provisions for a crew of six for one year, or the same amount of provisions for a crew of twelve for a trip of six months. In addition, the volume associate $d$ with the provisions for the original 600 day Mars su face excursion could either be maintained as backup ( $r$ traded for other cargo. A potential significant mass ccntributor not included in the original Mars Habitat was shielding for galactic cosmic rays and solar flares. Its mass impact projection remains premature. 


\section{Table 3: Payload System Mass Properties}

\begin{tabular}{lr}
\hline Life support & 12 \\
Crew accommodations & 45 \\
Health care & 5 \\
Primary structure & 20 \\
EVA & 8 \\
Electrical power distribution & 1 \\
Communication and data management & 3 \\
Thermal control & 4 \\
Spares/growth/margin & 7 \\
Science & 2 \\
Crew & 1 \\
- Total (mt) & 108 \\
\hline
\end{tabular}

The two story payload system included primary structure, at least two docking mechanisms mounted at the top and side, power distribution systems, life support system, consumables (oxygen, water, food, etc.), stowage, waste management, communication, science instruments/experiments, and fitness/recreation materials. It was a zero gravity concept with no provision for artificial gravity due to the less than one year trip time. The original Transit Habitat was designed to be functional in $3 / 8$ gravity after it separated and landed on the Martian surface.

\section{Table 4: Payload System Power Usage}

\begin{tabular}{|c|c|}
\hline Life support & 12. \\
\hline Thermal contract system & 2.2 \\
\hline Galley & 1. \\
\hline Logistic module & 1.8 \\
\hline Airlock & 0.6 \\
\hline Communications & 0.5 \\
\hline Personal quarters & 0.4 \\
\hline Command center & 0.5 \\
\hline Health maintenance facility & 1.7 \\
\hline Data management system & 1.9 \\
\hline Audio/Video & 0.4 \\
\hline Lab & 0.7 \\
\hline Hygiene & 0.7 \\
\hline SC/Utility power & 5. \\
\hline Total (kWe) & 29.4 \\
\hline
\end{tabular}

The scaled-up total mass of the crew payload was $108 \mathrm{mt}$ (twice that of the original design). Table 3 illustrates the payload mass properties. The primary contributor was crew accommodations $(45 \mathrm{mt}$ ) of which $\sim 80 \%$ were consumables. Other major items were primary structure $(20 \mathrm{mt})$ and the life support system $(12 \mathrm{mt})^{11}$. Table 4 provides the nominal power usages. The leading contributor was the life support system $(12 \mathrm{kWe})$. The total power requirement for the payload was $30 \mathrm{kWe} "$.

Defining and engineering the interface between the payload and the vehicle represents one of the most important, time consuming, and complex tasks in preparing for launching today's space missions. In advanced concepts such as this, the payload interface is somewhat ambiguous. The structural attachment (adapter) was a simple truss assembly of negligible mass. Interface hardware (auxiliary power connections, sensors, etc.) was also of negligible mass.

\section{CENTRAL TRUSS}

The primary structure linking the major systems of the vehicle was chosen to be a truss network in order to minimize mass while retaining strength. The light weight truss material was Aluminum Graphite/Epoxy (Al GrEp). This material and the strut/joint/node design was adopted from a tested, earlier concept for the International Space Station. The truss cross section was changed from a square to a hexagon in order to accommodate the six propellant tanks. A structural strut from a prior design was used, where its length had been reduced from $5 \mathrm{~m}$ to $3.5 \mathrm{~m}$, yet the overall cross-bracing arrangement (one diagonal per section) was retained. Figure 7 illustrates one truss section. Table 5 contains the mass properties of a single section, where the struts were scaled based on uniform lengths and the connecting hardware mass properties were maintained ${ }^{12}$. Despite a central truss length of $-225 \mathrm{~m}$ (requiring 65 sections), the total mass was only $5 \mathrm{mt}$. Accommodating axial and lateral loading, however, necessitated modest redesign.

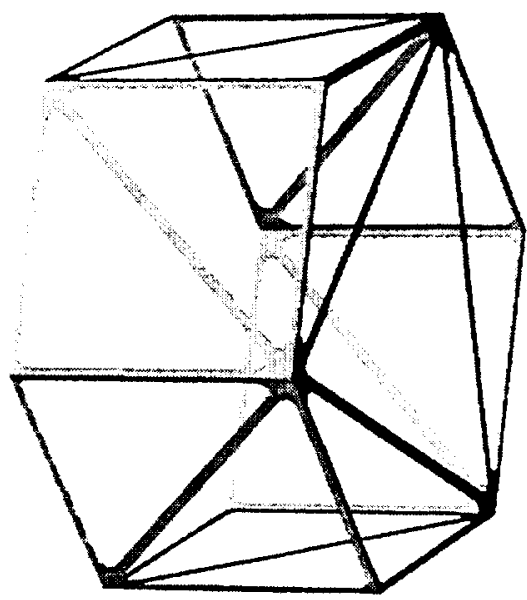

Figure 7: Central Truss Section 
The axial loading was assumed to be greatest at the aft end, where the propulsive thrust would have to be applied to the vehicle. A separate thrust structure was designed to take the $5,567 \mathrm{lb}_{\mathrm{f}}$ thrust load and distribute it axially into the six longitudinal struts. Its mass was minimal, with the 18 truss struts of radii 1.36 times greater than the baseline.

\section{Table 5: Central Truss Section Mass Properties}

\begin{tabular}{lr}
\hline Longerons $(6+6)$ & 21 \\
Diagonals $(6)$ & 15 \\
Diagonal on hexagonal face $(3)$ & 5 \\
Strut end joint $(2 * 19)$ & 20 \\
Node joint half $(2 * 19)$ & 13 \\
Nodes $(6)$ & 2 \\
\hline Total $(\mathrm{kg})$ & 76 \\
\hline
\end{tabular}

Given the low bending moment limit of the struts (scaled from the original $5 \mathrm{~m}$ strut cantilever failure at $\left.1,024 \mathrm{ft}^{\mathrm{l}} \mathrm{b}_{\mathrm{f}}\right)^{12}$, the lateral loads were of more concern. The radiators with a mass of $59 \mathrm{mt}$ each, at a average total moment arm of $75 \mathrm{~m}$, had to be attached at each node and at each strut midpoint to reduce the bending moment to a level comparable to the scaled limit value. This case was of greatest concern at the maximum acceleration, 1.514 milli-g's, encountered at end of mission. The other systems produced lower moments due to lower masses and/or small moment arms. Ample attachment hardware was needed and its mass was conservatively estimated as twice that of the entire central truss network. Thus, the total mass of the central truss network was $15 \mathrm{mt}$

\section{FUSION REACTOR SYSTEM}

\section{Plasma Modeling and Characteristics}

Modeling of the plasma conditions was performed through a 1-D plasma power balance computer program ${ }^{13}$. It was designed to analyze generic, small and large aspect ratio, tokamak fusion reactors, inductive and non-inductive heating, driven and ignited operation, burning DT, DD, or $D^{3} \mathrm{He}$ fuels. By pursuing peaked temperature and number density profiles within the core of a plasma, a relatively small fusion producing region was established, satisfying Lawson and ignition criteria without necessitating large beta $(\beta)$ throughout the plasma. The lower temperature and number density outer regions would contribute to a volume-averaged $\beta$ value within MHD stability limits.
This approach is tremendously attractive for space propulsion applications where compact size, thus reduced mass, is of paramount importance. Profile shape factors $(\delta)$ for temperature $(T)$, number density $(\mathrm{n})$, and current density $(\mathrm{J})$ were of the functional form given in equation (4), and were integrated along the minor radius ( $r$ ) (where $0 \leq r \leq a)$ with a concentric ellipse approximation used.

$$
\begin{gathered}
x(r)=<x>f(r) \quad \text { where } \\
x=(T, n, J) \\
f(r)=\left(1+\delta_{T, n, J}\right)\left(1-r^{2} / a^{2}\right)^{\delta_{r, J . J}} \\
x=\frac{2}{a^{2}} \int_{0}^{a} x(r) r d r
\end{gathered}
$$

Charged particle and neutron power density, including $\mathrm{DD}$ side reactions, were integrated as functions of number density and radius (along with temperature dependent reactivities). Bremsstrahlung and synchrotron radiation power densities were also integrated as functions of temperature and number density (and thus radius). From these quantities, other primary reactor characteristics such as plasma current, magnetic field, confinement time scaling, etc., were solved for while satisfying constraints such as critical beta and plasma power balance. Volume averaged quantities such transport power and radiation loss were used to determine initial available charged power for propulsicn and available waste power for conversion to auxiliary electrical power respectively. Plasma transport loss power, which included convection and conduction loss, represented the primary source of fusion reaction charged products for propulsion application and thus the quantity to be maximized.

$D^{3} \mathrm{He}(1: 1$ ratio) was chosen as the reactor fuel in order to maximize the charged transport power output and minimize neutron output power fraction. It was decided that in the time frame of this concept, reactor c peration at a plasma temperature of $50 \mathrm{keV}$ would $r$ spresent only an incremental technological challeng! over that of a DT-based concept operating at $10 \mathrm{keV}$ (and a fuel significantly more conducive to space propulsion application). Also, as was discussed earlier, solar system-class operation presupposed propellant and fuel supply availability in the hydrogen and helium-rich outer planet atmospheres and satellites, mitigating supply issues surrounding ${ }^{3} \mathrm{He}$. 
Table 6: Selected Reactor and Plasma Characteristics

\begin{tabular}{lc}
\hline Major radius (m) & 2.48 \\
Minor radius (m) & 1.24 \\
Aspect ratio & 2.0 \\
Elongation & 3.0 \\
Plasma volume $\left(\mathrm{m}^{3}\right)$ & 225.8 \\
Safety factor (edge) & 2.50 \\
Safety factor (axis) & 2.08 \\
Fuel ion density $\left(10^{20} / \mathrm{m}^{3}\right)$ & 5.0 \\
Electron density $\left(10^{20} / \mathrm{m}^{3}\right)$ & 7.5 \\
Plasma temperature $(\mathrm{keV})$ & 50 \\
Volume averaged beta & 0.318 \\
Confinement time (sec) & 0.552 \\
Average neutron wall load $\left(\mathrm{MW} / \mathrm{m}^{2}\right)$ & 1.03 \\
Average radiation wall load $\left(\mathrm{MW} / \mathrm{m}^{2}\right)$ & 5.20 \\
Ignition margin & 1.235 \\
Toroidal magnetic field (centerline) $(\mathrm{T})$ & 8.9 \\
Maximum magnetic field (coil surface) $(\mathrm{T})$ & 52.1 \\
Gain factor $(\mathrm{Q})$ & 73.1 \\
Plasma current $(\mathrm{MA})$ & 66.22 \\
Bootstrap current fraction & 0.934 \\
Wall reflectivity & 0.98 \\
Number density profile shape factor & 1.0 \\
Temperature profile shape factor & 2.0 \\
\hline
\end{tabular}

$D^{3} \mathrm{He}$ fuel with a spin vector polarized parallel to the magnetic field was used to capitalize on the up-to $50 \%$ enhancement in fusion reactivity cross section ${ }^{14,15}$, tremendously improving the charged output power. The methods of creating and utilizing polarized fuel have been developed through theory (DT and $D^{3} \mathrm{He}$ ) and experiment (DT) ${ }^{14,15}$. Although much work remains on maintaining fuel polarization, it is well known that ionization and atomic collision processes cannot result in depolarization ${ }^{15}$. However, there are potentially significant design impacts to the first wall, neutral beam injection, and fuel injection that will be discussed later.

Table 6 illustrates many other reactor and plasma characteristics. The total energy confinement time $(\tau)$ was $0.552 \mathrm{sec}$ and was in good agreement with the 1992 International Thermonuclear Experimental Reactor (ITER) $\mathrm{H}$-mode scaling law $(0.565 \mathrm{sec})$ that is a function of plasma current $\left(I_{p}\right)$, toroidal magnetic field $\left(B_{T}\right)$, number density $(n)$, total plasma heating power $\left(P_{L}\right)$, average atomic mass $(A)$, major radius $(R)$, minor radius (a), and elongation ( $\mathrm{K}$ ) (equation (5)) ${ }^{16}$.

$$
\tau=0.021 I_{p}^{0.55} B_{T}^{0.91} n^{0.17} P_{L}^{-0.55} A^{0.5}(R / a)^{-0.19} R^{2.3} \kappa^{0.7}
$$

A somewhat shorter confinement time was obtained from the 1997 ITER $L$-mode scaling law ${ }^{17}$ of $\tau=0.216$ sec. The primary driving terms in the $\tau$ scaling were found to be the large values of plasma current, applied magnetic field, total plasma heating power, and major radius. Although it is not clear which sub-ignited, experimental database-derived scaling law would be more representative of ignited plasma conditions for a propulsion system, it was reasonable to conclude that the proposed concept was consistent with what is currently known.

The critical beta $\left(\beta_{\text {crit }}\right)$ constraint was satisfied by requiring the total plasma current to be (equation (6)):

$$
I_{p}=\beta_{c r i t} a B_{T} / \beta_{N}
$$

where the Troyon coefficient $\left(\beta_{\mathrm{N}}\right)$ of 0.05 (a somewhat greater than typical value $\left(0.035<\beta_{\mathrm{N}}<0.04\right)$, based on a recent analytic study ${ }^{18}$ ) was used for a low aspect ratio tokamak. The large $\beta_{\text {crit }}$ and magnetic field required for space propulsive applications suggested that great leverage existed with maximizing $\beta_{\mathrm{N}}$ in order to minimize large $I_{p}$ and its impact on reactor design.

A density weighted, volume averaged plasma $T$ of $50 \mathrm{keV}$ and an $n_{i}$ of $5 * 10^{20} \mathrm{~m}^{-3}$ were chosen. Representative $n$ and $T$ profile shape factors of 1.0 and 2.0 respectively were chosen to enhance fusion power production.

$$
\begin{aligned}
I_{\text {hoot }} & =2.86^{*} 10^{-4} f(v) a \sqrt[4]{\frac{a}{R}} \\
& {\left[\left(\frac{1+\kappa^{2}}{2}\right)\left(n\left(1.55 T_{e}+T_{i}\right)\right)\right]^{-0.5} }
\end{aligned}
$$

The significantly greater fusion product $n T \tau$ for a $D^{3} \mathrm{He}$ fueled reactor led to the requirement for large plasma current $(\sim 66 \mathrm{MA})$ in order to obtain sufficient confinement. To provide for this current, a diffusion-driven bootstrap current, first experimentally observed in large tokamaks, was relied upon. The large $T_{i}$ and $n_{i}$ present in the core of $D^{3} \mathrm{He}$ fueled reactors could benefit from significant, arbitrarily $(90 \%+)$ large $e^{19}$, bootstrap fractions, where only modest seed currents provided by external heating would be needed. A $93 \%$ bootstrap current $\left(I_{\text {boot }}\right)$ was used based on equation (7), derived from neoclassical transport theory of tokamaks ${ }^{20} . f(v)$ was set equal to 0.3 to approximate the $T_{i}$ and $n$ radial profile shape factors consistent with 
the 1-D model used. Equation (7) is valid so long as the bootstrap fraction is above $75 \%$ and safety factor is greater than 2 for MHD-stable operation. With a large $I_{\text {boot }}$ of $\sim 62 \mathrm{MA}$, only a small seed current $\left(\mathrm{I}_{\text {seed }}\right)$ of $\sim 4$ MA was necessary (equation (8)).

$$
I_{\text {seed }}=I_{p}-I_{\text {boot }}
$$

An injection power $\left(\mathrm{P}_{\mathrm{inj}}\right)$ of $108 \mathrm{MW}$, based on the estimated $^{21}$ current drive efficiency $\left(\gamma_{n b}\right)$ of $0.75^{*} 10^{20}$ $\mathrm{A} / \mathrm{Wm}^{2}$, and an electron number density $\left(\mathrm{n}_{\mathrm{e}}\right)$ of 7.5 $* 10^{20} / \mathrm{m}^{3}$ was calculated from equation (9).

$$
P_{i n j}=\frac{n_{e} R I_{\text {seed }}}{\gamma_{n b}}
$$

A sufficiently high $\beta(\sim 30 \%)$ was chosen to efficiently use the strong magnetic field and reduce synchrotron losses. An edge safety factor of 2.5 was chosen. A high wall reflectivity of 0.98 was chosen to mitigate synchrotron power loss.

An ignited reactor mode of operation was chosen, in addition to high bootstrap current, in order to minimize the re-circulating power fraction required and the concomitant conversion system mass for generating injection power. It was thought that a continuously thrusting propulsion system would be better served by this mode of reactor operation, where charged transport power was maximized and used exclusively for propulsior: purposes.

\section{Power Generation and Utilization}

Figure 8 illustrates the power input, output, and utilization. Of the 7,895 MW of fusion power produced, $96 \%$ was in the form of charged particles with the remainder in (largely $2.45 \mathrm{MeV}$ ) neutrons. Total power input, which included $108 \mathrm{MW}$ of injection power, was 8,003 MW, yielding a Q-value (fusion power/injected power) of $>73$. More than $3 / 4$ of the power out of the reactor $(6,145 \mathrm{MW})$ was charged transport power (D and $\mathrm{He}$ ions, protons, and electrons) used solely for direct propulsion. Radiation in the form of Bremsstrahlung (1,016 MW) and synchrotron (535 $\mathrm{MW}$ ) power, along with neutron power ( $307 \mathrm{MW}$ ) and other waste heat was used to produce $400 \mathrm{MW}$ of electrical power.

The choice of fuel and design of some of the reactor components was intended to maximize the fraction of useful power-out while minimizing the

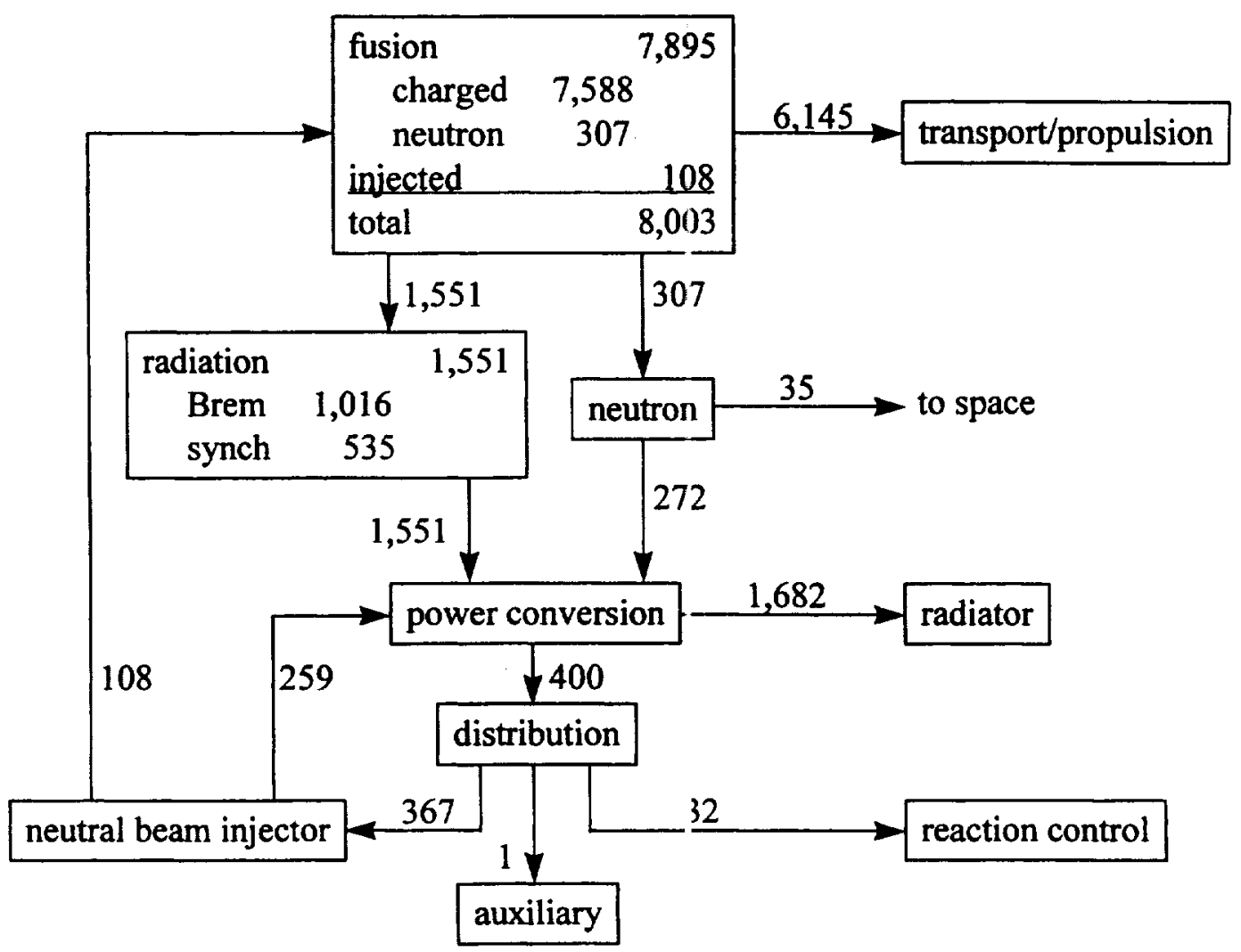

Figure 8: Power (MW) Input, Outp at, and Utilization 
fraction of power lost to (and the mass devoted to managing) unrecoverable radiation. This permitted the dedication of all charged power to propulsion, while scavenging only waste Bremsstrahlung and synchrotron radiation from the first wall to produce electrical power through a heat cycle. Since the electrical power that could otherwise be produced at high efficiency was in excess of requirements, the reactor was deliberately designed to permit as much of the neutron radiation as possible to escape directly to space ( $35 \mathrm{MW}$ ). Electrical power requirements were dominated by the negative ion neutral beam injector (N-NBI) at $367 \mathrm{MW}$ which provided the $108 \mathrm{MW}$ of injection power needed for current drive. Most of the remaining power was needed by the arcjet reaction control system. All other auxiliary power requirements were two or more orders of magnitude lower and included superconducting coil refrigeration, propellant tankage refrigeration, fuel injector, start/restart battery recharge, communications, avionics, and crew operations. All waste power was rejected via the radiator arrays $(1,682 \mathrm{MW})$, with the RCS power (32 MW) exhausted/radiated to space.

To sustain a total fusion power of $7,895 \mathrm{MW}$, $D^{3} \mathrm{He}$ fuel with a specific energy of $3.52 * 10^{14} \mathrm{~J} / \mathrm{kg}$ must be consumed at a rate of $\sim 22 \mathrm{mg} / \mathrm{sec}$. The fraction of fuel burn up $\left(f_{b}\right)$ was estimated from equation (10), where $\langle\sigma v\rangle$ is the Maxwellian averaged fusion reactivity for $D^{3} \mathrm{He}$ fusion reaction $\left(0.536<\left(<\sigma v>10^{-22}\right.\right.$ $\left.\mathrm{m}^{3} / \mathrm{sec}\right)<1.726$ for $\left.50<\mathrm{T}_{\mathrm{i}}(\mathrm{keV})<100\right)$.

$$
f_{b}=\left[1+\frac{2}{n \tau<\sigma v>}\right]^{-1}
$$

The average $D^{3} \mathrm{He}$ burn up fraction was estimated to be only $1 \%$, requiring a fuel mass flow rate $\left(\dot{m}_{\text {fuel }}\right)$ of 2.2 $\mathrm{g} / \mathrm{sec}$. At this consumption rate, a 235 day trip to Saturn required $45 \mathrm{mt}$ of $\mathrm{D}^{3} \mathrm{He}\left(27 \mathrm{mt}\right.$ of $\left.{ }^{3} \mathrm{He}\right)$. Note that $\sim 18$ $\mathrm{kg} / \mathrm{yr}$ represents a rough estimate of the potential U.S. annual production capability of ${ }^{3} \mathrm{He}^{22}$.

\section{Reactor Components}

A small major radius $(2.48 \mathrm{~m})$, small aspect ratio (2.0) device was chosen to minimize size and mass. The scaling for $\beta$ favored elongated $(3: 1)$, compact devices with large plasma currents ( $66 \mathrm{MA})$ and moderately large centerline magnetic fields $(8.9 \mathrm{~T})$. These magnetic field requirements led to very large toroidal field (TF) coil currents (9.2 MA). Twelve coils were used to generate the toroidal magnetic field; seven poloidal field (PF) coils were used to provide the necessary plasma stability.

Figure 9 illustrates the radial build, including an upper half of one TF "D-shape" coil. Beginning with the major axis centerline, a $7.35 \mathrm{~cm}$ radius annulus provided a flow channel for the slush hydrogen propellant. A $21.8 \mathrm{~cm}$ thick Titanium alloy provided structural support for the current-induced coil structural loads. Twelve $13 \mathrm{~cm}$ diameter high temperature superconducting TF coils carried the currents to generate the toroidal magnetic field. The cylindrical LiH blanket, $45 \mathrm{~cm}$ thick, protected most of the central core from the $2.45 \mathrm{MeV}$ neutrons. A toroidal shell of gaseous helium (GHe), $23 \mathrm{~cm}$ thick at $25 \mathrm{~atm}$, served as the heat transfer fluid for the first wall, which was made of a high strength (at very high temperature) Molybdenum alloy. The first wall's heat was due to average neutron and radiation loadings of 1 and 5 $\mathrm{MW} / \mathrm{m}^{2}$ respectively. The helium flowed through toroidal shells of the Molybdenum alloy that were 0.5 $\mathrm{cm}$ (outer) and $0.75 \mathrm{~cm}$ (inner) thick. A scrape off layer of ash was assumed to be $10 \%$ of the minor radius, with the minor radius being $1.24 \mathrm{~m}$. The outer radial dimensions were the same, except for a slightly thicker blanket $(50 \mathrm{~cm})$ to better attenuate the neutron flux.

The cross-section of the TF coils is illustrated in figure 10 . The coils were $75 \%$ by area $\mathrm{YBa}_{2} \mathrm{Cu}_{3} \mathrm{O}_{7}$ (YBCO, a high temperature superconductor) surrounded by a helium refrigerant, followed by an aluminum lithium outer shell. YBCO was chosen for its extremely high critical current density at moderate to large external magnetic fields, even at temperatures as high as $77 \mathrm{deg} \mathrm{K}^{23}$. These high densities permitted greatly reduced central coil radii compare to Type II superconductors, more efficient use of central core volume for neutron attenuation, and permitted incorporating a central propellant flow channel. The minimum coil radius was calculated by setting the sum of all magnetic field components from the other coils ${ }^{24}$ (including that of the far end of the same coil) equal to an empirically derived equation for critical current density $\left(J_{\text {crii }}\right)$ as a function of external field $\left(B_{e x}\right)^{23}$. The result was equation (11), where $I_{c}$ is the current in a single TF coil, $N$ is the number of coils, $R_{1}$ and $R_{2}$ are the distances from the major axis to the centerline of the inner and outer TF coils respectively, $r_{c}$ is the radius of the TF coil, $f_{c}$ is the cross sectional area fraction carrying current in the TF coil, $\mu_{0}$ is the permeability of free space, and $A$ and $B$ are the curve fitted exponent and scale factor of the exponential empirical equation of YBCO J $\mathrm{J}_{\text {crit }}$ vs. $\mathrm{B}_{\text {ext }}$. 


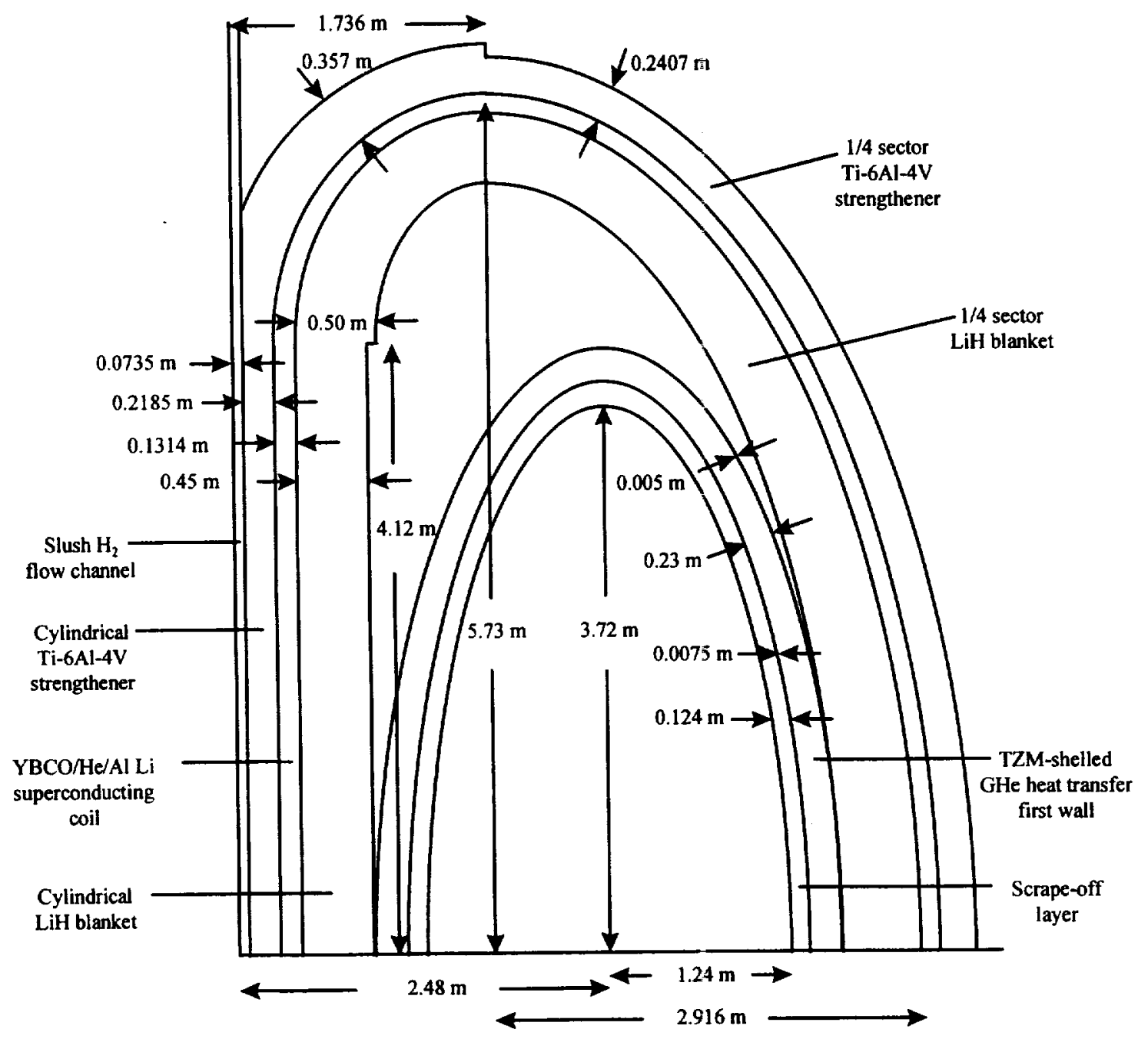

Figure 9: Reactor Radial Build

The limiting $\mathrm{B}_{\text {ext }}$ at the coil was $20.4 \mathrm{~T}$ with a corresponding $r_{c}$ of $6.57 \mathrm{~cm}$. The total field at the coil (self plus external) was $48.4 \mathrm{~T}$; though as will be explained later, the coils were centrally repositioned, driving the final field at the coil to $52.1 \mathrm{~T}$. The $\mathrm{J}_{\text {crit }}$ was conservatively evaluated at $77 \mathrm{deg} \mathrm{K}$, although the conductor was actually cooled to $70 \mathrm{deg} \mathrm{K}$.

The load carrying assessment was made by utilizing a reasonably accurate $(99 \%+)$ approximation for sizing constant tension D-shape magnets for fusion reactors ${ }^{24}$. By calculating the force per unit length $\left(f_{1}\right)$ given by equation (12) (where radius of curvature angle $(\phi)$ and conductor radius $\left(c_{r}\right)$ ) then substituting into equation (13) defining the radius of curvature $(\rho)$ of a flexible conductor under constant tension $(T)$, the differential equation (14) could then be solved describing the geometric shape of the coil ${ }^{25}$ (where $\mathrm{z}$ is the axi: of toroidal symmetry).

$$
\begin{aligned}
\left.\frac{\mu_{0} I_{c}}{2 \pi} \cdot \frac{N}{R}\left[1+\frac{1}{\left(\frac{R}{R_{1}}\right)^{N}-1}+\frac{1}{\left(\frac{R_{2}}{R}\right)^{N}-1}\right]-\frac{1}{\left(R-R_{1}\right)}\right] \\
-\frac{1}{A} \operatorname{Ln}\left[\frac{I_{c}}{f_{c} \pi r_{c}^{2} B}\right]=0
\end{aligned}
$$




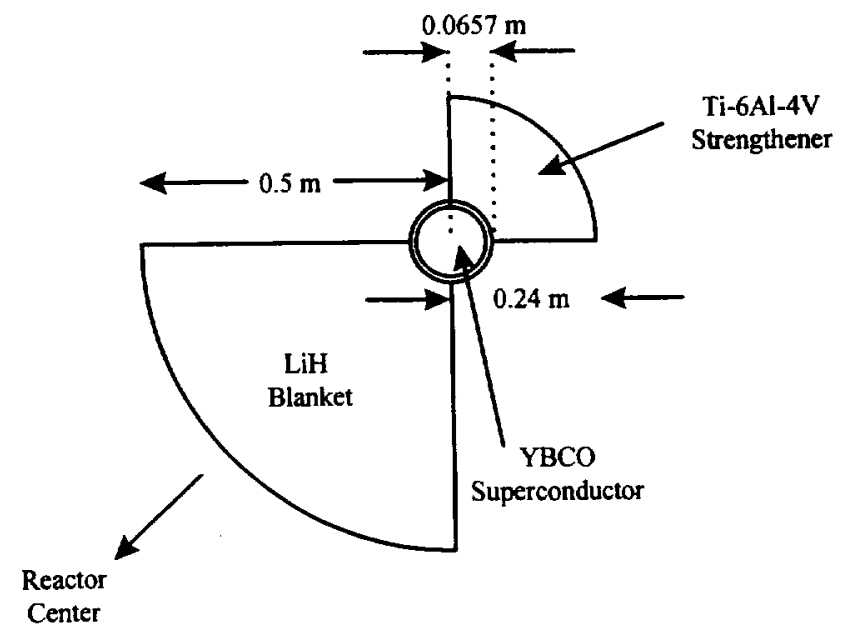

Figure 10: TF Coil Cross-Section

$f_{l}=\frac{\mu_{o} N I^{2}}{4 \pi R}\left[1+\frac{1}{N}\left(\cos \phi+\frac{R}{\rho} \ln \frac{1.284 R}{c_{r} N}\right)\right]$

$$
\begin{gathered}
\rho_{l}=\frac{T}{f_{l}} \\
\rho_{l} \frac{d^{2} \rho}{d z^{2}}= \pm \frac{\mu_{0} N I_{c}}{2 \pi}\left[1+\left(\frac{d \rho}{d z}\right)^{2}\right]^{3 / 2}
\end{gathered}
$$

The geometry of the D-shaped TF coils were determined by curve fitted polynomials to the solutions of equations (12) through (14) of the form of equation (15). Equation (15) was parameterized for four essential coil dimensions $\left(\mathrm{P}_{\mathrm{i}}\right)$, where the outermost coil location $\left(\rho_{2} / R_{2}\right)$ for the $\phi=0$ position was set as the independent variable.

$$
\frac{P_{i}}{R_{2}}=\sum_{j=0}^{4} A_{j}\left(\frac{\rho_{2}}{R_{2}}\right)^{j}
$$

The radial build resulting from equations (15) produced a design with an excessive $R_{1}$ (for the $H_{2}$ propellant channel and central strengtheners) and insufficient volume for the cylindrical $\mathrm{LiH}$ blanket. As a result, $\mathbf{R}_{\mathbf{l}}$ was decreased and the other primary dimensions were adjusted accordingly to maintain the general $\mathrm{D}$ shape. The tension loads, however, were recalculated for the actual TF coil positions for consistency, even though this produced greater loads and thus required more massive strengtheners. In addition, both vertical dimensions had to be increased $50 \%$ to accommodate the large elongation (3.0) of the reactor design. The original algorithm (equation (12)) was not found to be conducive to highly elongated toroids. The end result of this approach to sizing and placing TF coils may not have been rigorously correct, but was reasonably self consistent and accounted for the primary current-driven tension forces on the TF coils.

The high current (9.2 MA) carried in the coils that produced the tremendous tension loads necessitated strengtheners made from the highest yield strength material suitable for cryogenic applications. A Titanium alloy, Ti-6Al-4V, was selected for its high yield strength $\left(19 * 10^{8} \mathrm{~N} / \mathrm{m}^{2}\right.$, solution treated and aged ELI) even at 32 deg $\mathrm{K}^{26}$. The Ti-6Al-4V cross section design chosen was that of a $1 / 4$ sector cylinder (figure 10 ), mounted onto the outer (opposite the reactor core) TF coil surface, to efficiently carry the tension load with minimum mass. This design was used for the strengtheners of the top circular and outer elliptical coil sections, while a cylindrical design was used for the strengthener of the inner linear coil section to more efficiently share the greatest loads. Despite its enormous yield strength, the thickness of the outer and top $1 / 4$ sector cylinder strengtheners, and inner cylinder strengthener were calculated to be $24.07 \mathrm{~cm}, 35.7 \mathrm{~cm}$, and $21.85 \mathrm{~cm}$ respectively. The strengtheners were the second greatest massive structures in the entire vehicle. The attractive Ampere's law-driven forces between the TF coils were also calculated but were found to be less than $6 \%$ of those of the tension loads. Cross-bracing for these as well as the "overturning" forces were included in the design, with the minor additional mass penalty assumed to be accounted in the PF coils. The total 12 TF coil mass was calculated to be $221 \mathrm{mt}$.

The $\mathrm{LiH}$ neutron blanket also used a $1 / 4$ sector cylinder design so that the coil would be protected from neutron radiation no matter where in the plasma the neutrons were created. The orientation of the blanket was directly facing the reactor core (i.e. on the opposite side of the coil from the strengtheners, figure 10). This design minimized blanket mass while also permitted neutrons that were not impacting the coils to pass directly to space. This less than $4 \pi$ shielding design meant that human operations could not be conducted everywhere around the reactor without protection while 
the reactor was operating. However, the convergence of the blankets at the forward (crew module-facing) and aft (magnetic nozzle-facing) poles of the reactor would provide sufficient protection for human operations within certain angular constraints. A minimum $50 \mathrm{~cm}$ blanket thickness was recommended for $D^{3} \mathrm{He}$ fueled reactors $^{27,28}$. Packaging constraints within the central core driven by structural loading required that the $\mathrm{LiH}$ shielding there be reduced to $45 \mathrm{~cm}$. This increased neutron activation/heating and remains an issue to be resolved. The calculated $\mathrm{LiH}$ blanket mass was $56 \mathrm{mt}$.

Over $88 \%$ of the $307 \mathrm{MW}$ of neutron power was intercepted by the $\mathrm{LiH}$ blankets; the balance of the power was permitted to escape into space. The neutron heating consisted of $92 \mathrm{MW}$ of power into the cylindrical blanket and $180 \mathrm{MW}$ of power into the $1 / 4$ sector blankets. This heat was removed by supplemental GHe from the heat transfer system used at the first wall. Though not shown on figure 9 , a $3 \mathrm{~cm}$ diameter $\mathrm{GHe}$ channel adjacent to the coils within the $\mathrm{LiH}$ was included in the GHe first wall thickness to provide the heat transfer mechanism. This heat was assumed to be of comparable quality to the other waste heat in the system. An attractive alternative would be to examine if the space facing blanket sides would be able to re-radiate heat to space as gray bodies while remaining within material thermal limits. Initial geometric analysis indicated that the arrangement of the blankets was such that most neutron radiation was intercepted in the volumes immediately forward of and aft of the reactor, thereby protecting the vehicle systems from activation and structural damage.

Minimal analysis was done on the PF coils. Based on ITER and similar test reactors, seven PF coils were assumed, with coil and shielding designs and masses scaled to $60 \%$ of the TF coils as recommended by similar advanced small aspect ratio reactor studies ${ }^{21}$. The cross sectional makeup was assumed to be identical to the TF coils, including strengtheners, but without LiH blankets, since much of the PF coils would be shadowed by the TF coil blankets. The total $7 \mathrm{PF}$ coil mass was calculated to be $133 \mathrm{mt}$.

The thickness of the first wall radiation shield and GHe pressure vessel was based on evaluation of the pressure and energy deposition within the structure. Neutron and Bremsstrahlung/synchrotron radiation wall loadings were 1.03 and $5.2 \mathrm{MW} / \mathrm{m}^{2}$ respectively. These values are representative of other power reactor designs for first walls and divertors. To accommodate these loadings, the molybdenum alloy "TZM" (Mo-0.5Ti$0.1 \mathrm{Zr}$ ) was selected based on its suitability for very high temperature applications such as radiation shields. TZM is also expected to provided high synchrotron radiation reflectivity, which is mandatory to facilitate its re-absırption into the plasma. TZM's high yield strength of $62 \mathrm{MPa}$ (at $1650 \mathrm{deg} \mathrm{C}$ ) ${ }^{29}$ with a mass density half that of tungsten, made it a good choice to withstand the circumferential stress due to the $25 \mathrm{~atm}$ pressure of $\mathrm{GHe}$ heat transfer fluid within the pressure vessel. The initial thickness based on stress limits $(0.5$ $\mathrm{cm}$ ) had to be increased for the inner (first wall) to permit sufficient absorption of the estimated $50 \mathrm{keV}$ gamma radiation. Using an extrapolated mass absorption coefficient ${ }^{30}\left(\mu_{\mathrm{a}} / \rho\right)$ for molybdenum of 2.22 $\mathrm{cm}^{2} / \mathrm{g}$, the required shield thickness $(x)$ was estimated from equation (16):

$$
P_{o u t}=P_{i n}\left(\frac{a+\Delta s+x}{a+\Delta s}\right) e^{-\left(\frac{\mu_{n}}{\rho}\right) \rho x}
$$

A thickness of $0.75 \mathrm{~cm}$ was sufficient to reduce the radiation power $\left(\mathrm{P}_{\mathrm{in}}\right)$ to under $100 \mathrm{~W}\left(\mathrm{P}_{\text {out }}\right)$, where $\Delta s$ was the scrape off thickness and $\rho$ was the Mo mass density. The mass of the first wall/pressure vessel was calculated to be $18 \mathrm{mt}$. In a related issue, TZM could have a detrimental effect on spin polarized fuel. It has been suggested from today's research reactors that recycling of fuel off a metallic first wall surface could cause significant depolarization ${ }^{15}$. This remains an issue to be resolved should this effect prove to be true.

A $7.35 \mathrm{~cm}$ radius channel through the center of the reactor was provided for the thrust augmentation mass of the slush hydrogen propellant. Although some propellan: warming was expected to take place, the primary reason for channeling the propellant in this way was io align the bulk $(96 \%)$ of the propulsive mass flow velocity and downstream magnetic nozzle thrust vectors. As will be discussed, this approach has the potential to cause adverse impacts to reactor operations.

\section{Reactor Fueling}

The preponderance of fuel injector design, fabrication, and operation experience has been with ${ }^{2} \mathrm{D}$, and DT fuelling. Two stage, light gas gun devices have accelerated $1-3 \mathrm{~mm}$ frozen ${ }^{2} \mathrm{D}$ pellets up to $2.5 \mathrm{~km} / \mathrm{sec}$ 31, 32. Alternate concepts such as electrothermal guns have been tested with plastic (Lexan polycarbonate) pellets to similar velocities ${ }^{33}$. The gross dimensions and masses fir most concepts were small compared to other major sy: tems ( $<1 \mathrm{~m}$ lengths and $<1 \mathrm{mt}$ masses) ${ }^{34,35}$.

Very little effort, however, has be devoted to issues surrounding injection of frozen $D$ pellets encapsulating (liquid) ${ }^{3} \mathrm{He}$. It is thought that injecting such pellets during ignited, high temperature operation will be very difficult ${ }^{15}$. An earlier conceptual design 
study of a mirror reactor propulsion concept suggested a two stage mechanical (centrifugal) and electromagnetic railgun injector be used for $D^{3} \mathrm{He}$ pellets $^{36}$. This study, as well as others, suggested a potential approach involving frozen light metal shelled (lithium deuteride) pellets encapsulating ${ }^{3} \mathrm{He}$ fluid ${ }^{15,}{ }^{36}$. Estimated final velocities of up to $30 \mathrm{~km} / \mathrm{sec}$ with acceleration lengths limited to up to $20 \mathrm{~m}$ forced one study to use light metal shelling to mitigate barrel heating and stress on the pellet. The light metal shelling did have a detrimental effect ( $17 \%$ loss in jet power $)^{36}$ on reactor performance, even at small $(<6 \%)$ pellet mass fraction. Pellet evaporation, however, is presently not thought to be a major potential contributor to fuel depolarization ${ }^{15}$.

Given the paucity of experimental data, it was hypothesized that a $\mathrm{D}^{3} \mathrm{He}$ fuel pellet injector would be no greater than $1 \mathrm{mt}$ in mass (including injector, feed lines, knife, piston), with a modest $(<5 \mathrm{~kW})$ power requirement. The ample central truss length should permit a sufficiently low pellet acceleration so as not to require light metal shelling. Tankage mass for the $D^{3} \mathrm{He}$ fuel was scaled from the slush hydrogen propellant storage system. The $45 \mathrm{mt}$ of fuel required a $3 \mathrm{~m}$ radius cylindrical tank, $17 \mathrm{~m}$ long and a "wet" mass (including reserves, residuals, and losses) of $7 \mathrm{mt}$.

\section{Divertor}

By diverting the outer plasma layers to the propulsion system, fusion energy served to directly heat propulsive mass without first going through intermediate, material heat flux-limited, low-efficiency power conversion equipment. An efficient divertor thus enabled a direct thrust approach to space propulsion by transferring high energy charged particles out of the reactor and into the reservoir of the magnetic nozzle. As was shown, $99 \%$ of the particles exiting the reactor will be un-reacted $\mathrm{D}$ and ${ }^{3} \mathrm{He}$ ions and electrons. The balance of the particles (neutral impurities, ${ }^{4} \mathrm{He}$, and $\mathrm{p}$ ash) must be kept to low densities to maintain good confinement and maximum propulsion performance. As these particles cooled and migrated to the outer field lines in the scrape-off layer, they eventually were pulled into the divertor and out of the reactor. Plasma $T$ and $\mathrm{n}$ in the scrape-off were calculated to be $\mathrm{T}<4 \mathrm{keV}$, and $\mathrm{n}_{\mathrm{i}}<1.95 * 10^{20} / \mathrm{m}^{3}$ (figure 11 ).

The divertor's geometric design (single null, located in the reactor aft) was patterned somewhat after ITER's ${ }^{37}$. Three PF-like divertor coils (carrying reduced current through a smaller coil radius) were within the reactor's minor radius, between the TF coils to divert the magnetic field outside of the reactor. The

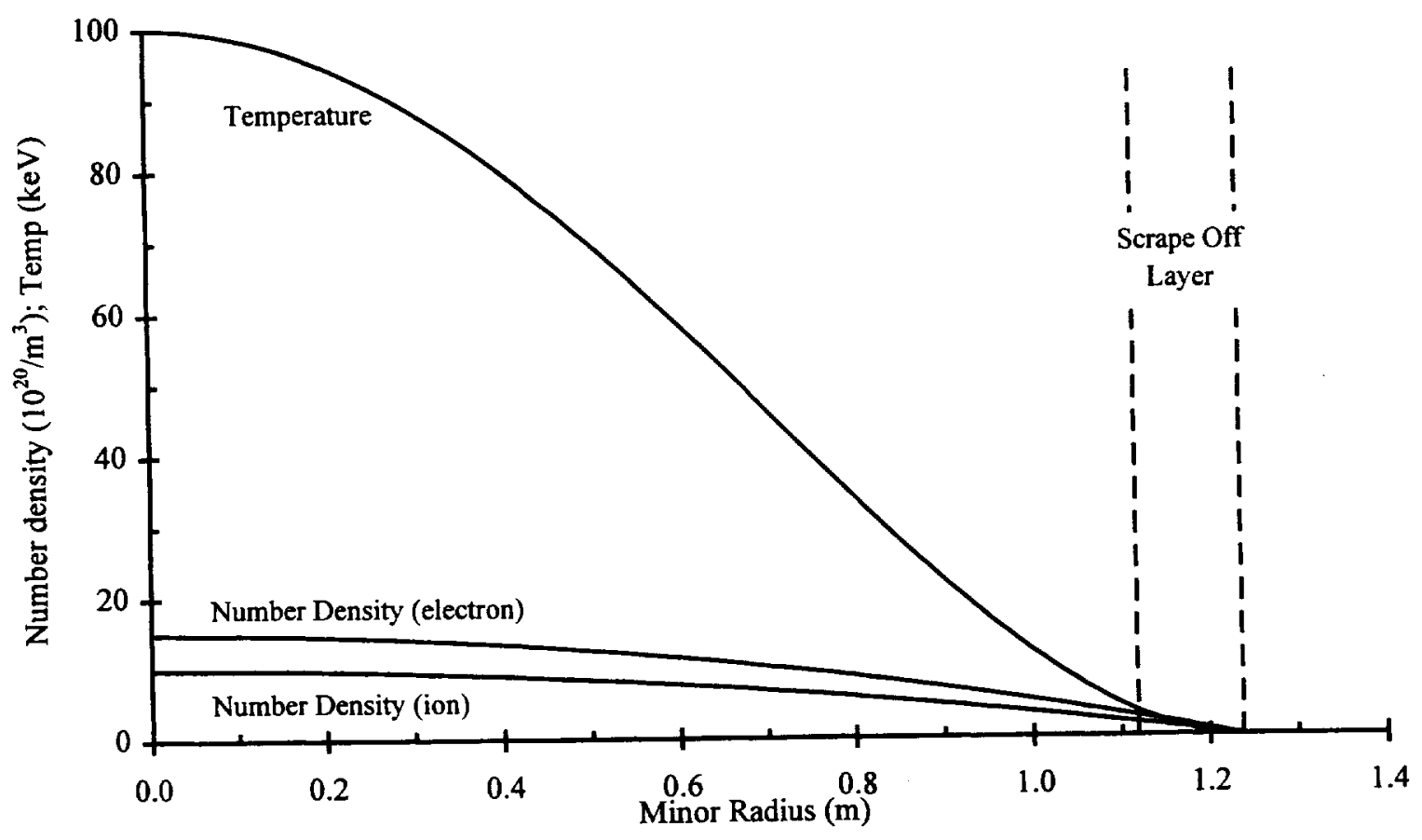

Figure 11: Reactor Plasma Temperature and Number Density Radial Profiles 
field lines exited and re-entered the reactor through the double annulus, carrying the exhaust plasma to the magnetic nozzle. The estimated combined width of the double annulus and middle coil was $0.5 \mathrm{~m}$, scaled from ITER geometry ${ }^{38}$. The divertor coils' plasma facing material must be able to withstand high temperatures and high erosion rates. Carbon fiber composites, such as Aerolor $\mathrm{A} 05^{39}$, are likely candidates to tolerate surface temperatures up to $1,500 \mathrm{deg} C$ with peak heat loads of at least $5 \mathrm{MW} / \mathrm{m}^{2}$ and transient heat loads potentially as great as $20 \mathrm{MW} / \mathrm{m}^{2}{ }^{37}$. Total mass of all three coils plus additional system mass (structure, power lines, etc.) was set to $4 \mathrm{mt}$.

The divertor's operational concept was fundamentally different than ITER's or any of those used in today's experimental reactors. Today's reactors are not designed to exhaust vast quantities of transport power, operate in steady state, accommodate immense wall loading without assistance of radiation from injected inert gas, or exhaust plasma without collision with a target. In addition, today's divertor structural designs and resulting masses are driven by requirements that are not expected to be a primary issue with a space propulsion concept. ITER's divertor consists of 60 cassettes weighing $25 \mathrm{mt}$ each $^{37,39,40}$. Their mass is attributed to both withstanding the immense structural loadings initiated by disruptions and also shielding the vacuum vessel from neutron radiation ${ }^{37}$. It was assumed that steady state operation of the reactor concept would at least minimize if not eliminate disruptions altogether. And since there will be no vacuum vessel, the other reason was mute. As a result, the entire divertor was envisioned as a series of TZM structures covered with Aerolor A05 plasma facing material protecting the divertor coils. The coils were assumed to be the same cross-sectional design as the TF/PF coils, although at a much lower, to be determined current density. Since the divertor coils were inboard of the TF coils, a difficult task awaits in designing them to be of sufficient field strength to extract plasma scrape off without having a detrimental affect on the primary field and the fusion process. Resolution of this problem is fundamental to the feasibility of this concept.

\section{MAGNETIC NOZZLE}

The conversion of the reactor's transport power into directed thrust was accomplished by the magnetic nozzle in two steps. In the first step, the nozzle mixed high enthalpy transport plasma from the divertor with the injected hydrogen propellant in order to reduce the excessive temperature and increase total charged propellant mass flow. In the second step, it converter. the propellant enthalpy into directed thrust by accelerating the flow through diverging magnetic field lines. In addition, its magnetic field prevented the high temperature plasma from coming in contact with the nozzle's coils and structural members that make up the thrust chamber. Thus for a fully ionized flow, the lines of magnetic flux served as the containment device, minimizing heat transfer loses and the need for actively cooled structure.

The $I_{s p}$ 's of 20,000 to $50,000 \mathrm{lb}_{\mathrm{f}} \mathrm{sec} / \mathrm{lb}_{\mathrm{m}}$ and corresponding $\alpha$ 's required for multi-month travel to the outer planets required ion reservoir temperatures $\left(T_{\text {res }}\right)$ of 100 's $\mathrm{eV}$. As was discussed, the too great temperature and too small number density plasma layers that entered the diverter had to be adjusted prior to acceleration through the nozzle so as to produce the mission appropriate $I_{s p}$. This was accomplished by heating/ionizing slush hydrogen thrust augmentation propellant by the escaping reactor plasma, thereby producing the desired values of bulk plasma temperature (thus $I_{s p}$ ) and mass flow rate (thus thrustto-weight). The propellant was preheated by its pass through the reactor core with residual heat from absorbed neutron radiation. The propellant was then injected into the reservoir along the nozzle centerline.

The "reservoir" of the magnetic nozzle was somewhat analogous to a conventional liquid chemical rocket engine's combustion chamber. Adjacent to the reactor's divertor, it consisted of two small radius superconducting coils of the same design and materials as the $\mathrm{TF}$ coils. Forming an "effective" $10 \mathrm{~cm}$ radius solenoid, they provided the meridional magnetic field to confine the converging propellant and reactor plasma streams until their temperatures equilibrated. The reservoir was in large part a "virtual" chamber due to its mostly skeletal design where magnetic field lines defined the flow boundary for charged particles passing through. This design minimized mass and heating concerns but also placed a premium on rapid, effective ionization and enthalpy equilibration (i.e. neutrals were lost through the sides). The second small radius coil also constituted the "throat" for the nozzle, where choked fow (sonic) conditions existed. An arbitrarily larger current loop radius third coil in the downstream, diverging section provided additional curvature to the magnetic field. The entire length of the assembly was somewhat arbitrarily set to $12 \mathrm{~m}$ with a total mass estimated at $4 \mathrm{mt}$. The nozzle coils were considerably less mas:ive than the TF/PF coils due to negligible neutron $r$ idiation, significantly lower required magnetic field (thus lower coil current and structural loads), and lightweight Al-GrEp composite structure. The entire layout, including number of coils, geometric and magnetic field curvature, was largely notional and was 


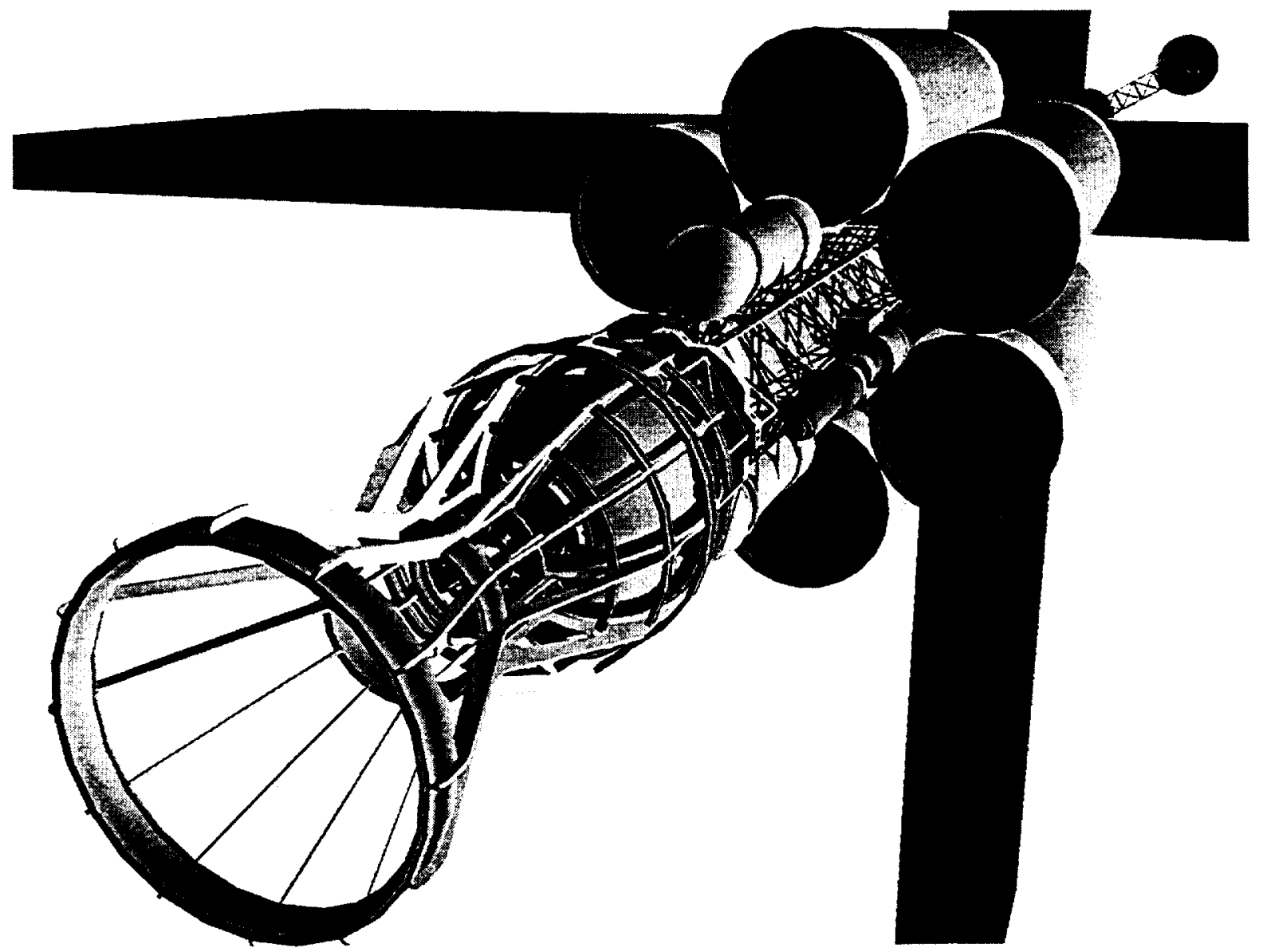

Figure 12: Magnetic Nozzle and Power Conversion System

intended to foster discussion, analysis, and experimentation into its salient aspects. Until mature design concepts with experimental data are available, magnetic nozzle designs, operations, and $\eta$, for fusion propulsion will be largely speculative. Figure 12 illustrates the layout of the magnetic nozzle.

Much of the potential jet power could be lost unless the internal energy can be efficiently converted into accelerated axial flow. Estimates of the relative importance of $\eta_{J}$ on piloted interplanetary travel have been recently reported '. It has been shown that low $\eta_{J}$ is particularly detrimental to payload mass fraction, since decreased $\eta_{\mathrm{J}}$ (at constant thrust) significantly increased propellant consumption. An example is illustrated in figure 13 for a case where $c / V_{c}=0.7$, representative of a fast, piloted outer planet mission. Although at a greater payload ratio than the design concept $(17.7 \%$ for a $100 \%$ efficient nozzle), the payload ratio is seen to be a strong function of $\eta_{\mathrm{J}}$, vanishing at an $\eta_{j}$ less than $50 \%$. Thus efficient magnetic nozzle designs appear to be essential for the class of systems envisioned for fast interplanetary travel, where $\eta_{\mathrm{J}} \leq 70 \%$ may not be tolerable.

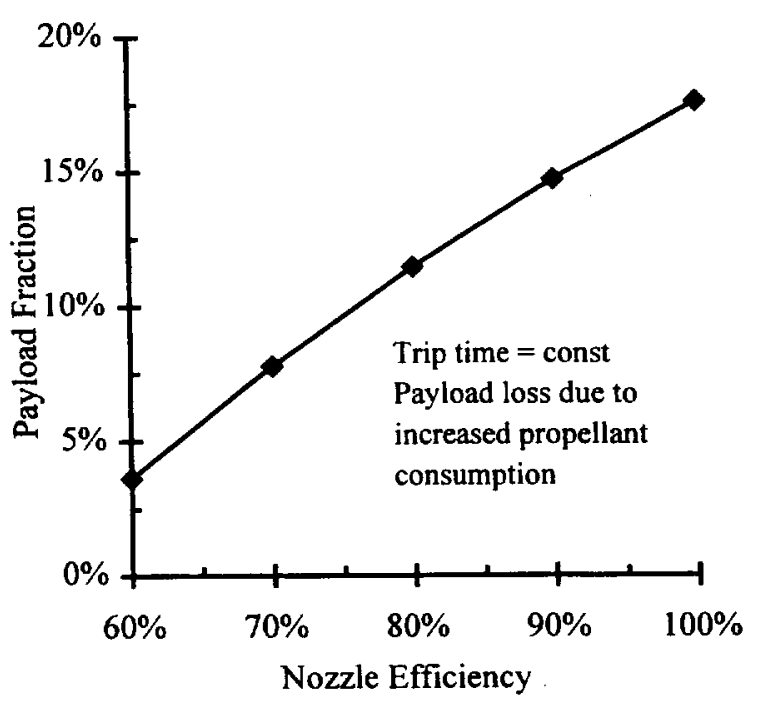

Figure 13: Nozzle Efficiency vs. Payload Fraction 
Magnetic nozzle experimentation applicable to fusion-class propulsion has been recently initiated ${ }^{41}$ by a team at NASA LeRC, Ohio State University (OSU), and Los Alamos National Lab (LANL). A series of three small, proof of concept experiments are focused on providing key experimental data for nozzles intended for GW jet power level, quasi-steady plasma flows. The first of these experiments is focussed on how the plasma would detach from the magnetic lines of force upon exiting a magnetic nozzle and produce net thrust. This problem lacks both theoretical and experimental definition and may represent the leading obstacle towards developing a practical fusion propulsion system. Of primary importance is obtaining plasma number density (thus mass flow rate) and velocity distribution data as functions of axial and radial position downstream of the throat. By integrating these quantities, $P_{j e t}$ and $F$ can be calculated, inferring a value for $\eta_{J}$. A variety of operating parameters should be investigated, such as reservoir temperature $\left(T_{\text {res }}\right)$, density $\left(\rho_{\text {res }}\right)$, magnetic field strength $\left(B_{\text {res }}\right)$, choice of propellants, and others. Initially using helium (eventually hydrogen) ions to mimic the exhaust plasma, these experiments will enable estimation of a nozzle's performance that can be correlated to fusion propulsion systems. Further experimentation will include designing and testing a rudimentary sub-scale magnetic nozzle similar to the one used for this concept, as well as designing and testing propellant injection for thrust augmentation. Until experimental data is available to correlate theoretical models, the performance of direct plasma-propelled fusion propulsion systems will be to a considerable extent conjecture. Some theoretical analysis projecting and defining their operation is available however.

The theory of how a magnetic nozzle would operate and the primary obstacles anticipated in its development were the subjects of a lengthy analysis ${ }^{42}$. Idealized 1-D, MHD-modified fluid dynamic equations for a magnetic nozzle with an applied meridional magnetic field were derived in that work. Various simplifying assumptions were made such as neglecting charged particle-neutral collisions, assuming a fluid (as opposed to a kinetic) model, and neglecting various loss mechanisms such as transport, radiative, resistive, and Hall effect. A simple, single turn coil was assumed to form the throat of the magnetic nozzle. Using this approach, along with other supporting analysis ${ }^{43,44.45}$, the flow conditions at key points through the nozzle were estimated. Isentropic flow was assumed throughout, with an arbitrary nozzle efficiency of $80 \%$ assumed. For simplicity, it was assumed that the $20 \%$ nozzle loss took place at propellant-fuel enthalpy transfer.
The required downstream conditions for the exiting fl $\mathrm{sW}\left(\eta_{\mathrm{J}} \alpha=3.136 \mathrm{~kW} / \mathrm{kg}\right.$ and $I_{\mathrm{sp}}=40,485 \mathrm{lb}_{\mathrm{f}}$ $\mathrm{sec} / \mathrm{lb}_{\mathrm{m}}$ ) were used to initiate the flow calculations. By working back up through the nozzle, the required fuel entry conditions from the divertor were found. These conditions were required to match the transport power available from the reactor. The 1-D model revealed that the propellant exit velocity $\left(V_{e x}\right)$ was approximately twice the throat (sonic) velocity $\left(\mathrm{V}_{\text {throat }}\right)$, shown in equation (17) ${ }^{42}$.

$$
I_{s p}=\frac{V_{e x}}{g_{c}}=\frac{2 V_{\text {throut }}}{g_{c}}
$$

The related throat state variables (where $T_{\text {throat }}, \rho_{\text {throat }}$, $(P R)_{\text {throat }}, B_{\text {throat }}$ and $A_{\text {throat }}$ are the temperature, density, pressure, magnetic field, and cross-sectional area in the throat, and $R$ is the gas constant using monatomic hydrogen (1.0078 AMU)) are given by equations (18) through (21):

$$
\begin{gathered}
T_{\text {throat }}=\frac{V_{\text {throat }}^{2}}{\gamma R} \quad \text { (18) } \\
(P R)_{\text {throat }}=\frac{F}{2 \gamma A_{\text {thraat }}} \quad(19) \\
\rho_{\text {throat }}=\frac{(P R)_{\text {throat }}}{R T_{\text {throat }}} \quad(20) \\
B_{\text {throat }}=\sqrt{2 \mu_{o}(P R)_{\text {throat }}} \quad(\text { with } \beta=1)
\end{gathered}
$$

The total thrust (F) was previously calculated to be $5,567 \mathrm{lb}$. An "effective" $10 \mathrm{~cm}$ throat radius was created by the magnetic field lines, with the structural throat ralius at $2 \mathrm{~m}$. The $10 \mathrm{~cm}$ radius was chosen so that the throat cross sectional area power flux of $1.56^{*} 10^{5} \mathrm{MW} / \mathrm{m}^{2}$ was comparable to proposed NTR designs $\left(1.16^{*} 10^{5} \mathrm{MW} / \mathrm{m}^{2}\right)^{46}$ and within extrapolation of current cryogenic engines such as the Space Shuttle Main Engine $\left(5.14^{*} 10^{4} \mathrm{MW} / \mathrm{m}^{2}\right){ }^{47}$. A $\beta=1$ condition was assumed to calculate the magnetic field strength and required current for throat and upstream reservoir magnet coils. A specific heat ratio $(\gamma)$ of $5 / 3$ was used for a moulatomic gas of three degrees of freedom.

The reservoir conditions were solved using equations (22) and (23), and equations (20) and (21) with reservoir values ( $M$ is throat Mach number $\equiv 1$ ). The $\mathrm{T}_{\text {res }}$ was $329 \mathrm{eV}$, an order of magnitude lower than 


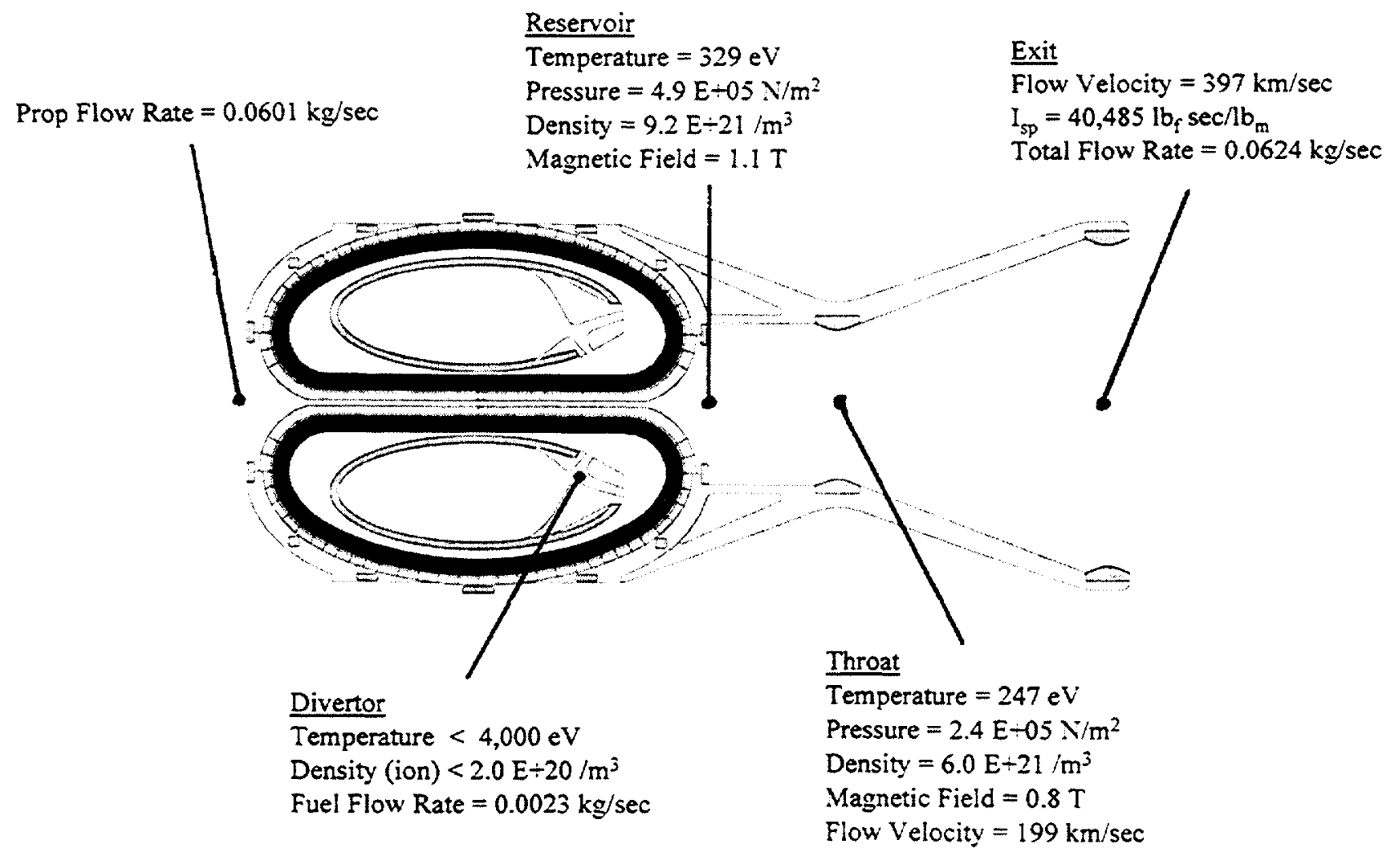

Figure 14: Plasma State Conditions Through Magnetic Nozzle

that at the scrape off boundary. The $\mathrm{B}_{\text {res, }}$ similar to $B_{\text {throat, }}$ was a comparatively modest $1.1 \mathrm{~T}$.

$$
\begin{aligned}
& T_{\text {res }}=\left[1+\frac{\gamma-1}{2} M^{2}\right] T_{\text {throat }} \quad(M=1) \quad(22) \\
& (P R)_{\text {res }}=\left[1+\frac{\gamma-1}{2} M^{2}\right]^{\frac{\gamma}{\gamma-1}}(P R)_{\text {throat }} \quad(M=1)
\end{aligned}
$$

The reservoir flow power $\left(\mathrm{P}_{\mathrm{res}}\right)$ and thermal velocity $\left(v_{\text {res }}\right)$, which are related by the stagnation enthalpy $\left(h_{\mathrm{res}}\right)$, can be estimated by equations (24) and (25). The flow power can then be related to the available transport power $\left(P_{\text {trans }}\right)$ from the reactor by equation (26). Thus the jet power and state conditions throughout the nozzle can be correlated to the power delivered to the reservoir by the divertor. The $20 \%$ loss associated with the enthalpy transfer was assumed to exit the nozzle in the form of neutrals, ions with velocity vectors not strictly aligned with the thrust vector, collisions with support structure, line radiation, charge exchange, and other losses. Figure 14 illustrates the plasma state variables through the nozzle.

$$
\begin{gathered}
P_{\text {res }}=\dot{m}_{\text {total }} h_{\text {res }}=\dot{m}_{\text {total }} \frac{\gamma}{\gamma-1} R T_{\text {res }} \\
v_{\text {res }}=\sqrt{2 h_{\text {res }}} \\
P_{\text {res }}=\eta_{J} P_{\text {trams }}=\eta_{J} \frac{1}{2} \dot{m}_{\text {fuel }} v_{\text {fuct }}^{2}=\frac{1}{2} \dot{m}_{\text {total }} v_{\text {res }}^{2}
\end{gathered}
$$

The augmentation propellant mass flow rate ( $\left.\dot{m}_{\text {prop }}\right)$ of $0.06 \mathrm{~kg} / \mathrm{sec}$ was easily found by subtracting $\dot{m}_{\text {fuel }}$ from $\dot{m}_{\text {total }}$. The augmentation propellant was $\sim 27$ times that of the fuel, emphasizing that a significant portion of the magnetic nozzle system must be dedicated towards accommodating the injection of augmentation propellant. This great a mass infusion into the flow stream (compared to the relatively low flow rate from the reactor) may be great enough to adversely affect the plasma conditions back into the reactor. This could represent a significant operational problem and warrants future assessment. 


\section{POWER CONVERSION}

The primary function of the power conversion system was to utilize the Bremsstrahlung and synchrotron radiation by "thermalizing" the energy flux so that it could be converted into electrical output power. A closed Brayton (gas) thermodynamic cycle was selected on the basis of proven design and fabrication experience, significant performance and reliability database, and the ability to use an inert gas (gaseous helium (GHe)) as the working fluid to transport heat energy (cooling the reactor first wall and TF coils) at high temperature (up to $2,000 \mathrm{deg} \mathrm{K}$ ) directly to the turbine.

As shown in the power flow diagram (figure 15), two sources of reactor radiated power of 1,823 $\mathrm{MW}$ were at $50 \mathrm{keV}$ and $2.54 \mathrm{MeV}$. In addition, 259 MW of waste heat from the N-NBI was also recycled as thermal energy. Thus from the total input heat of 2,082 $\mathrm{MW}, 400 \mathrm{MWe}$ of electrical power was produced for the N-NBI and other on board power requirements. The remainder of 1,682 $\mathrm{MW}$ was thermally radiated to space via the heat rejection system.
To neutralize gyroscopic torque generated by the power generating system, two $200 \mathrm{MWe}$ "turbosets" were used (figure 12), configured to rotate in opposite directions. The conceptual design of the power conversion system was patterned after helium turbine designs for nuclear power plants advanced in Germany three decades ago ${ }^{48,49}$. Some units, however, were actually constructed up to $25 \mathrm{MWe}$ power levels ${ }^{50}$.

The salient design and performance parameters for the two power conversion systems were regenerated cycles with $>80 \%$ effectiveness $\left(\varepsilon_{R G}\right)$ operating with a peak temperature (turbine inlet) of $1700 \mathrm{~K}$. The cycle temperature and pressure ratios of 2.52 and 2.15 , respectively, established the need for each unit to accommodate half the input heat 1,041 MW while generating the required output of $200 \mathrm{MWe}$. This set the required cycle efficiency at approximately $20 \%$. With the peak cycle pressure (i.e. compressor exit pressure) set at $25 \mathrm{~atm}$, the resultant inlet pressure was $11.7 \mathrm{~atm}$, and the total mass flow rate for each unit was $437 \mathrm{~kg} / \mathrm{sec}$.

A flat plate, square radiator design was chosen using light weight carbon-carbon, parallel duct heat

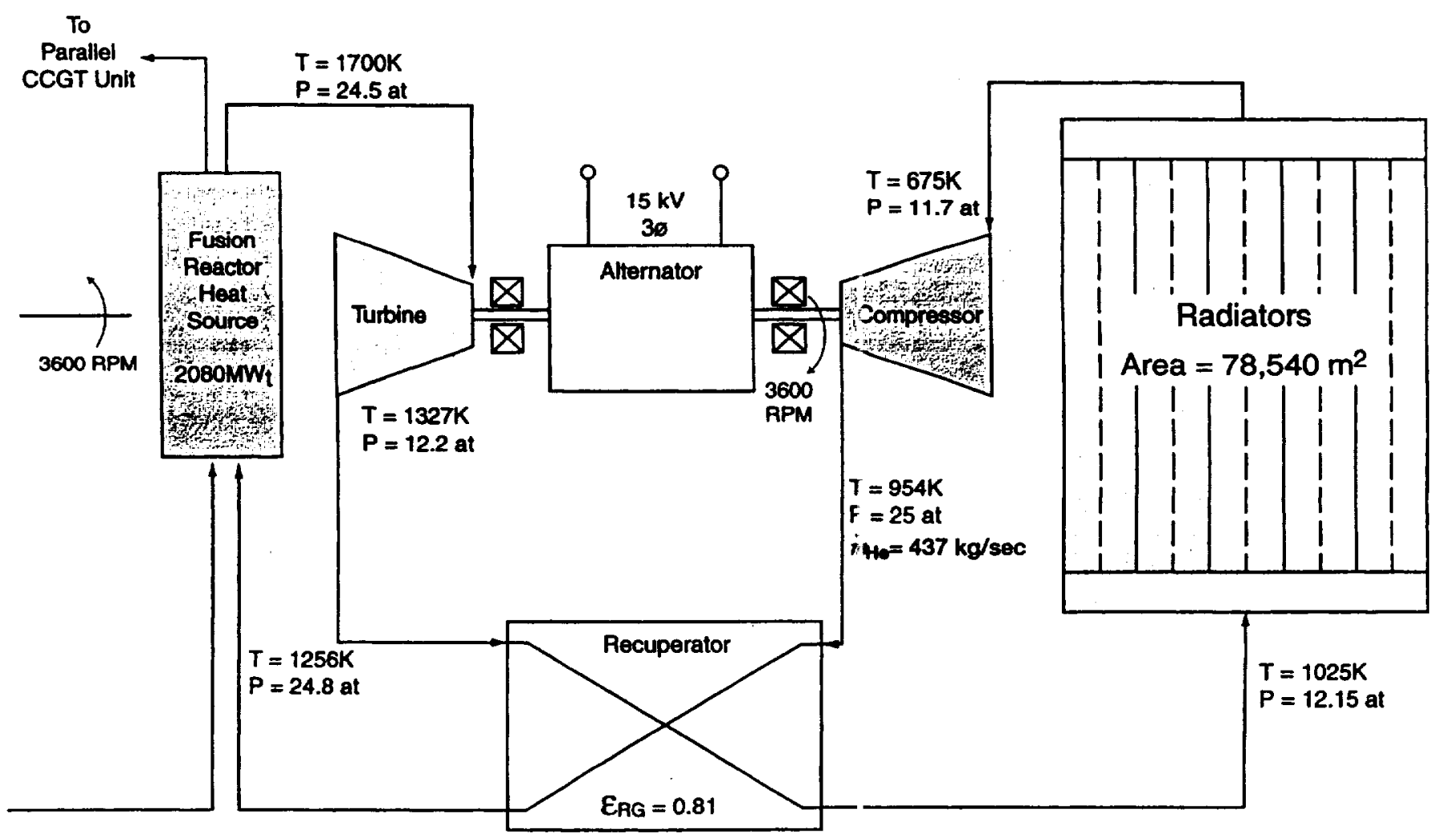

Figure 15: Single 200 MWe Closed Cycle Gas Turbine Power Conversion System 
pipes. This radiator technology has been proposed as the appropriate match for closed Brayton cycle with a high temperature gas reactor heat source ${ }^{51}$. The radiator parameters were derived from successful laboratory demonstration of specific mass up to $1.5 \mathrm{~kg} / \mathrm{m}^{2}$ (based on radiation from both sides), surface emissivity up to 0.9 , and radiating temperatures up to $650 \mathrm{deg} \mathrm{K} .{ }^{51}$ With GHe entering the radiator at $1,025 \mathrm{deg} \mathrm{K}$, the total radiating area for each of the turbosets was $78,540 \mathrm{~m}^{2}$, necessitating a radiating area of two $150 \mathrm{~m}$ square planar heat rejection surfaces, each rejecting heat from both sides. Thus for the two units, four radiating surfaces arranged in a "cruciform" configuration was required. Although this configuration resulted in radiative interchange between the surfaces, the overall view factor to space was only decreased from a value of 1 to 0.8 . The radiator area calculation has been adjusted to account for this penalty, as well as additional small waste heat dumps.

\section{Table 7: Power Conversion System Mass Properties}

\begin{tabular}{lr}
\hline Recuperator & 23 \\
Compressor & 20 \\
Turbine & 9 \\
Alternator & 23 \\
Conditioning & 40 \\
Radiator & 236 \\
Power conditioning radiator & 30 \\
\hdashline$-y^{-}-$ & 381 \\
\hline
\end{tabular}

The turboset casing diameters were $2.6 \mathrm{~m}$ and the length, including the alternator-generator were $\sim 18$ $\mathrm{m}$. With the turboshaft rotational speed of 3,000 to $3,600 \mathrm{rpm}$, the alternator could be driven at synchronous speed to generate 50 to $60 \mathrm{~Hz} \mathrm{AC}$ power. It is advantageous to generate most of the power at the voltage demanded by the largest loads. The power consumed by the N-NBI ( $367 \mathrm{MW}$ ) represented over $90 \%$ of the total power output of the power conversion system. As a result, the power would be generated in the appropriate form (voltage, amperage, phase) for the $\mathrm{N}$-NBI in order to minimize heavy power conversion transformers and associated systems. Almost $90 \%$ of the N-NBI power was required in the form of high voltage (3 steps of $150-170 \mathrm{kV}$ each) ${ }^{54}$ and respectable current $(64 \mathrm{~A})$. Voltage transformers, however, were included in the power management and distribution contribution to the total mass budget of $381 \mathrm{mt}$ for the power conversion system (Table 7).

\section{NEUTRAL BEAM INJECTOR}

Negative ion neutral beam injection (N-NBI) appears to be the most promising method of noninductive plasma heating, at high number density, due to its greater neutralization efficiency than positive ion NBI ${ }^{52}$. One of the leading, operational N-NBI is part of the Japan Atomic Energy Research Institute's (JAERI) JT-60U reactor. It was this N-NBI that was used as the basis for scaling a system capable of heating the non-bootstrap driven current of the design concept. The JT-60U N-NBI is capable of providing $500 \mathrm{keV}$ negative $D$ ions, at $\mathrm{n}=5^{*} 10^{19} / \mathrm{m}^{3}$, with a total beam power of $10 \mathrm{MW}^{52}$.

An injected power of $108 \mathrm{MW}$ was shown to be required from the N-NBI to heat the non-bootstrap (seed) current of $4.35 \mathrm{MA}$, an order of magnitude greater power than that delivered to the JT-60U. The system scaling approach taken was to retain the same system efficiencies, increase by an order of magnitude the input ion power from the ion sources, and adjust the N-NBI mass properties to reflect the space vacuum environment and additional ion source tanks.

To supply $108 \mathrm{MW}$ of neutralized $\mathrm{D}$ ion beam heating power, $367 \mathrm{MW}$ of power had to be supplied to the N-NBI. These considerable values resulted even after a deliberate effort was made to minimize the heating power required by maximizing the bootstrap current fraction. The various components of the N-NBI power consumption are illustrated in Table 8, and were scaled up from the JT-60U. Almost $90 \%$ of the power was needed by the acceleration power supply (315 MW), the system upon which the power conversion system would be designed to accommodate. The significant power losses were associated with the ion source tanks $(37 \%)$ and the neutralizer $(24 \%)$ and represent the majority of the waste heat $(259 \mathrm{MW})$ that was sent to the power conversion and rejection system.

\section{Table 8: Neutral Beam Injector Power Usage}

\begin{tabular}{lr}
\hline Cathode & 3 \\
Arc & 15 \\
Bias & $<1$ \\
Plasma grid filter & 2 \\
Extraction & 16 \\
Acceleration & 315 \\
Bending coil & 16 \\
\hdashline Total (MW) & 367 \\
\hline
\end{tabular}

One of the chief concerns in such a system scale-up was whether an order of magnitude increase in 
beamline power density could be achieved. The JT-60U configuration was thought to be able to accommodate a power density increase up to a factor of two and at most four ${ }^{33}$. Current research at JAERI on a $1 \mathrm{MeV} \mathrm{N}-\mathrm{NBI}$ device is focused on increasing current density to these levels $^{54}$. It is also pursuing a merged beam extractionacceleration system with a shorter length, smaller diameter, multiple channel neutralizer, enabling a more compact (thus less massive) design. It is anticipated that this research could eventually enable the N-NBI postulated for this design concept.

\section{Table 9: Neutral Beam Injector Mass Properties}

\begin{tabular}{|c|c|}
\hline Ion sources (20@2.5 mt each) & 50 \\
\hline Ion source steering mechanism & 1. \\
\hline Beam scraper & 0.6 \\
\hline Cryopumps & 1. \\
\hline Beam limiter & 0.4 \\
\hline Ion source tank & 1. \\
\hline Bellows & 0.6 \\
\hline Neutralizer & 0.5 \\
\hline Bending coils & 4. \\
\hline Ion dumps (positive $\&$ negative) & 1.2 \\
\hline Ion dump tank & 1. \\
\hline Calorimeter & 1.5 \\
\hline Beam limiter & 0.4 \\
\hline NBI bellows & 0.3 \\
\hline Isolation valve & 0.5 \\
\hline NBI port & 0.6 \\
\hline bellows & 0.2 \\
\hline Total (mt) & 64.8 \\
\hline
\end{tabular}

The total mass of the N-NBI was $65 \mathrm{mt}$. The $\mathrm{N}-\mathrm{NBI}$ mass properties are given in Table 9. A total of 20 ion source tanks (at $2.5 \mathrm{mt}$ each) dominate the $\mathrm{N}$ NBI mass. These $3 \mathrm{~m}$ diameter, $4.8 \mathrm{~m}$ high structures produce the $22 \mathrm{~A}, 500 \mathrm{keV}$ negative $\mathrm{D}$ ions by passing cesium through an arc discharge, then on through a transverse magnetic field to enhance negative $D$ ion yield, to a three stage multi-aperture extractoraccelerator that electrostatically increases the ion's energy to $500 \mathrm{keV}^{52,54}$. Its total mass was estimated by accounting for an order of magnitude increase in required ion yield coupled with deletion of structure associated with vacuum generation ${ }^{53}$. Other significant mass reductions from the JT-60U device included removal of hardware providing vacuum conditions in the ion source and dump tanks (from 25 to $1 \mathrm{mt}$ each), and length shortening-driven reductions of the neutralizer (from 6 to $0.5 \mathrm{mt}$ ) and NBI port (2 to 0.6 mt) due to test site physical layout rather than experimertal requirements ${ }^{19}$.

The N-NBI was placed within the aft central truss, adjacent to the reactor to shorten the beam line length. The entire system length was re-scaled to be approximately $32 \mathrm{~m}$. Injection of the neutral beam at right angles to the reactor plasma flow has the potential to depolarize spin polarized fuel ${ }^{15}$. To avoid excitation of magnetic fluctuations about whatever ion cyclotron frequencies might be encountered, one or more right angle turns in the beam line conduit may be required to mitigate this effect.

\section{PROPELLANT CRYO-TANKAGE}

The slush hydrogen propellant cryo-tankage was based on a pre-existing conceptual design', itself predicated on operational or previously designed conceptual liquid hydrogen propellant tanks ${ }^{55}$. The large quantity of propellant needed for the vehicle rendered even the largest past, current, or proposed liquid hydrogen tank designs unsuitable. The largest liquid hydrogen tank ever built and flown is the hydrogen component of the Space Shuttle's External Tank (ET), a $27.5 \mathrm{ft}(8.4 \mathrm{~m})$ diameter, $96.7 \mathrm{ft}(29.5 \mathrm{~m})$ long structure which can accommodate $103 \mathrm{mt}$ of propellant ${ }^{56}$. The Saturn V's S-II hydrogen tank, a $33 \mathrm{ft}$ $(10 \mathrm{~m})$ diameter, $53 \mathrm{ft}(16 \mathrm{~m})$ long structure accommodated $70 \mathrm{mt}$ of propellant ${ }^{57}$. In order to carry the significant quantity required for the design concept (1,292 mi), multiple tanks of an even greater capacity were obviously needed. Therefore, a conceptual design was made of the largest tank that appeared reasonable on the grounds of experience, engineering judgment, and ground transportation concerns. An existing conceptual design of liquid hydrogen tankage done in part by the co-authors' ${ }^{\prime}$ and based on an NTR vehicle concept $^{55}$, was adopted for slush hydrogen tankage. Support systems, net mass, and power required to accommodate slush hydrogen above and beyond those for liquid hydrogen were for the most part negligible ${ }^{58}$. One concern, however, was allowing sufficient additional tank volume and strength to accommodate the slush that would liquefy during launch and up to docking with refrigeration systems on the vehicle concept. Due to its ascent dependency and lack of launch s senario definition, no design provisions were made fo: this concern at this time. The original NTR tankage design was for a human Mars mission. A graphite-epoxy (GrEp) composite hydrogen tank material was used to obtain considerable mass savings over advanced aluminum alloys ${ }^{1}$. The $10 \mathrm{~m}$ diameter was mantained in order not to significantly impact manufacturing and ground transportation limits. The 
3:1 aspect ratio (cylindrical barrel section length-todiameter) yielded a total tank length of $37 \mathrm{~m}$. The net slush hydrogen propellant available for main impulse per tank was calculated to be $\sim 207 \mathrm{mt}$.

\section{Table 10: Cryo-Tankage Mass Properties}

Structure
upper ellipsoidal dome
cylindrical barrel section
lower ellipsoidal dome
forward skirt
ring
cylinder
aft skirt
$\quad$ ring
cylinder
insulation
Fluid system
feedlines and insulation
valves and manifolds
autogenous bleed
zero g vent
purge
Electrical and power system
avionics and power
instrumentation
telemetry
range safety
shielding
harnessing
Reaction control system
500 lbf (2) thrusters
50 lbf (24) thrusters
propellants
Interface hardware
OMV-derived interface
tank attach central truss

Total (mt)

Table 10 lists the subsystem masses for the designed cryo-tankage, which totaled to a dry mass of $22 \mathrm{mt}$. The subsystems were based on and modified from three studies, all of which were based primarily on the two Centaur upper stage configurations flying on the then-current Atlas/Centaur and Titan IV/Centaur expendable launch vehicles. In the resulting tank design, over $46 \%$ of the total mass was attributed to the composite tank (upper and lower domes, plus barrel section), and $22 \%$ attributed to the $5 \mathrm{~cm}$ multi-layer cryo-insulation plus aluminum micro-meteoroid shielding (sized for Mars orbit "mean space temperatures" to approximate the range of Earth to Saturn environment). A significant amount of mass and system complexity was required by considering the means for delivering the propellant to the departure orbit via an HLLV. The fully loaded propellant tankage must be launched with all the requisite ETO-related systems (avionics, telemetry, range safety, etc.). Upon separation from the HLLV, the cryo-tankage must be capable of controlling its own attitude, rendezvous, and dock with the vehicle concept, thus requiring a reaction control system and interface hardware.

\section{Table 11: Cryo-Tankage Mass Summary}

\begin{tabular}{llrr}
\hline Propellant & main impulse & 207 & \\
& flight perf. reserve & 2 & \\
& residuals/losses & 6 & \\
Stage Dry & & & 22 \\
Adapter & & 6 \\
Contingency (30\% of dry mass) & -15 \\
\hline GLOW (mt) & & 251 \\
\hline
\end{tabular}

Table 11 illustrates the mass summary for the fully loaded cryo-tankage in its ETO launch configuration (i.e., including adapter and associated contingency). Added to the useable, main impulse propellant was a flight performance reserve (FPR) of $1 \%$ (of main impulse propellant), consistent with past mission experience to accommodate in-flight dispersions. Estimates of residuals and chill-down losses were also included and made up 3\% (of the total tankable propellant). The adapter was sized to accommodate launch loads and was chargeable to the propellant tankage payload, although it would only be used from ETO and not retained on the interplanetary vehicle concept. A $30 \%$ weight growth allowance was assessed on the tankage dry mass and adapter. The gross liftoff weight (GLOW) of a fully loaded tankage payload would be $251 \mathrm{mt}$.

\section{REFRIGERATION}

Two Garret, reverse Brayton refrigeration systems with helium working fluid were used for cooling the propellant/fuel tankage and the TF/PF/divertor/magnetic nozzle superconducting coils. The first system used a $14 \mathrm{deg} \mathrm{K}$ low temperature sink $\left(T_{L}\right)$ to accommodate the slush hydrogen's boiling point. A second system was used to cool the YBCO superconductor in the coils, where $T_{L}$ was set to $70 \mathrm{deg}$ $\mathrm{K}$. Even though other higher temperature, more 
appropriate refrigeration systems (neon, nitrogen, etc.) might have been used for the coils, commonality with the propellant/fuel system was pursued for the sake of simplicity.

The electrical power $\left(\mathrm{P}_{\text {refrig }}\right)$ required to operate the system was calculated by equation (27), where the heat rejection temperature $\left(T_{H}\right)$ was chosen to be 350 $\operatorname{deg} \mathrm{K}$, and was only a weak function of the quantity of heat rejecting for the values of interest. The refrigerators' coefficient of performance (COP) was set at $15 \%$ of the Carnot COP, consistent with cooling loads above $500 \mathrm{~W}^{59}$. Using a Mars orbit heat flux to approximate an average Earth to Saturn thermal environment, and the tankage insulation and shielding described earlier, the total thermal power to be removed (Q) from the seven propellant/fuel tanks was $0.923 \mathrm{~kW}$. The heat to be rejected from the coils (produced by the neutron power flux intercepted by the blankets and not removed by the GHe heat transfer fluid, plus ancillary heating) was not explicitly calculated however. Instead, an arbitrary $500 \mathrm{~W}$ of residual heat was assumed to reach the outer aluminum-lithium coil surface. The total thermal power to be removed from the $12 \mathrm{TF}, 7 \mathrm{PF}, 3$ divertor, and 3 magnetic nozzle coils was $12 \mathrm{~kW}$. Thus the total electrical powers required for the tankage and superconducting coil refrigeration systems were $<0.15$ MWe and 0.32 MWe respectively.

$$
P_{\text {refrig }}=\frac{Q}{0.15}\left[\frac{T_{H}-T_{L}}{T_{L}}\right]
$$

The mass of the refrigeration $\left(\mathrm{M}_{\text {refrig }}\right)$ system was determined from equation (28) and included all subsystems except power source and radiator ${ }^{59}$. The additional radiator mass was determined to be small compared to the main array. Thus, the total refrigeration power (operation and rejected heat) to be rejected was merely added to the main array. The total masses for the tankage and superconducting coil refrigeration systems were 1.4 and $52.4 \mathrm{mt}$ respectively.

$$
M_{\text {refrig }}=91.9 * 10^{0.0468(\log (Q))^{2}}\left[\frac{T_{H}-T_{L}}{280}\right]
$$

\section{AVIONICS / COMMUNICATIONS}

The concept vehicle's avionics suite would be composed of primary/backup computers, guidance, navigation, and control system (GN\&C), tracking, data display, sequencing, and instrumentation. The exponential rate of growth in speed and capability of electronics and computer technology will no doubt enable future systems to be vastly superior to today's systems at only a fraction of the mass and power required. Thus minimal attention was devoted towards trying to extrapolate avionic system capabilities and requirements. A sufficient avionics suite of the future should be available with a total mass $<1 \mathrm{mt}$ with an arbitrarily small power consumption.

The communication system was presumed to be a derivative of the recently developed $\mathrm{Ka}$ band ( 20 to $30 \mathrm{GHz}$ ) technology. This NASA LeRC digital processing and storage communication technology is currently capable of integrated data, voice, and video with typical throughput rates in excess of $10^{8} \mathrm{bits} / \mathrm{sec}$ and up to $10^{10} \mathrm{bits} / \mathrm{sec}{ }^{60,61,62}$. Two $15 \mathrm{~m}$ diameter deployabie $\mathrm{Ka}$ band dish antennas were used for the audio, video, and data communication system. Dual antennas were used to enable two way simultaneous communications with the departing and arriving destination planets. Mounted on $18 \mathrm{~m}$ truss booms aft of the crew habitat payload, the dish antennas were positioned at $45 \mathrm{deg}$ angles with respect to the radiator arrays to minimize heating (figure 16).

The total avionics/communication system mass was set at $2 \mathrm{mt}$. The power for the avionics suite was assumed to be comparable to the Space Shuttle orbiter available power ${ }^{63} 0.02 \mathrm{MW}$. The power required by the communication antennas was indeterminate, since the planetary antennas' diameters and powers were undefined. Therefore a value was set at $0.2 \mathrm{MW}$.

\section{START/RE-START REACTOR \& BATTERY}

The startup system consisted of a $1 \mathrm{MWe}$ nuclear fission reactor power system and a nickel hydrogen $(\mathrm{NiH})$ bipolar battery bank. Weeks prior to departure, the startup reactor was used to gradually refrigerate the $\mathrm{TF} / \mathrm{PF} / \mathrm{div}$ ertor/magnetic nozzle coils, initiate and ramp up their current, provide auxiliary power, and charge-up the battery. When all systems and crew wese ready, $a \sim 10 \mathrm{sec}$ plasma startup sequence was initi ited with battery discharge providing the $\sim 1$ GJ of enc rgy and $100 \mathrm{MW}$ of power needed for startup.

?rior to current startup and neutral beam heating to ignition conditions, $\sim 1$ to $2 \mathrm{MW}$ of auxiliary radio-frequency (rf) heating at the electron cyclotron frequency was used to create a small volume of high conducti ity plasma $\left(\mathrm{T}_{\mathrm{e}} \sim 100 \mathrm{eV}\right.$ and $\left.\mathrm{n}_{\mathrm{c}} \sim 10^{19} / \mathrm{m}^{3}\right)$ outboard of the plasma major radius which assisted in the curre 1 t startup process ${ }^{64}$. This plasma conditioning, referred to as preheating, permitted a small radius $\left(a_{0} \sim\right.$ 0.2 to $0.4 \mathrm{~m}$ ) current channel to be established with a relatively low initial loop voltage $(<25 \mathrm{~V}$ as opposed to 


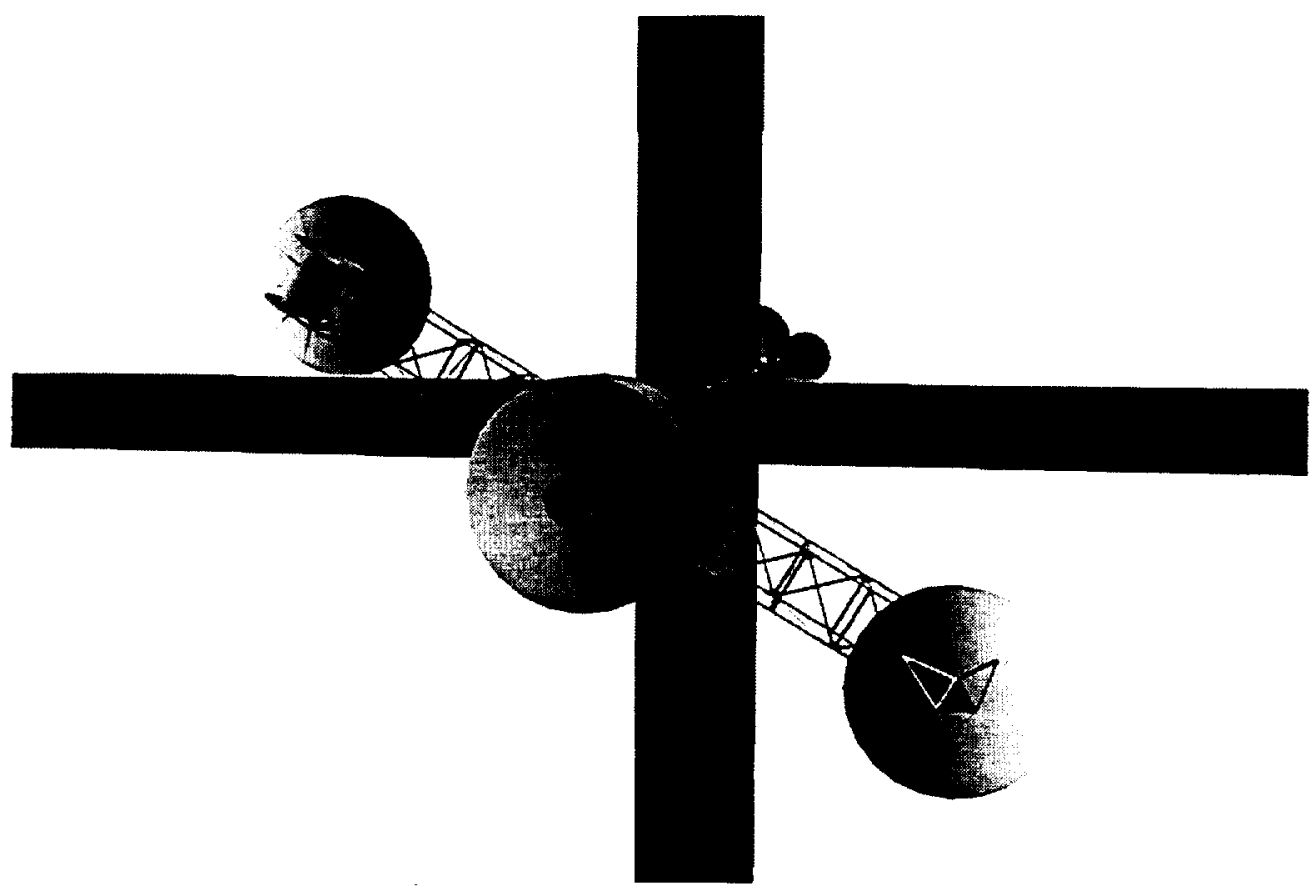

Figure 16: Dual Ka Band Antennas and Crew Payload

$\sim 100-200 \mathrm{~V}$ without $\mathrm{rf}$ assist). With the onset of current initiation and establishment of the desired safety factor $q$ in the small current channel, the startup major radius near the outboard midplane was gradually shifted inward to $R=2.48 \mathrm{~m}$. During this "expanding radius startup"6s, new layers of plasma were added to the warm core through ionization of a regulated gas feed. Major radius compression permitted minor radius expansion and a simultaneous increase in plasma current while a constant $q$ was maintained. As the plasma minor radius grew in size, sufficiently high levels of current and plasma density were achieved to ensure adequate confinement of energetic protons from $D^{3} \mathrm{He}$ fusion. Neutral beam heating to ignition conditions could then commence.

A high temperature gas cooled reactor (at $\sim 1$ MWe) power system would supply auxiliary/standby power during emergency re-start of the main propulsion system. The reactor heated $1.5 \mathrm{~kg} / \mathrm{sec}$ flow of $\mathrm{GHe}$ to $1,500 \mathrm{~K}$ in order to drive a gas turbine. Designed for minimum mass, total system was estimated at $<5 \mathrm{mt}$.

The NiH bipolar batteries were derivatives of devices designed and tested at NASA LeRC. These $82 \%$ efficient, high peak power systems were capable of specific energies of $180 \mathrm{~kJ} / \mathrm{kg}$ and energy packaging densities of over $80 \mathrm{Whr} / \mathrm{liter}^{66,67}$. For the required startup energy pulse, a bank of volume $3.36 \mathrm{~m}^{3}$ with a mass of $5.6 \mathrm{mt}$ would be needed. State of the art high energy capacitor-batteries ${ }^{68}$ were also considered, but despite their attractive energy densities, their specific volumes were not competitive with $\mathrm{NiH}$ batteries.
Table 12: Power Usage Summary (Nominal and Re-start Battery Re-charge)

\begin{tabular}{|c|c|c|c|c|}
\hline$\overline{\mathrm{NBI}}$ & & & & 367. \\
\hline RCS & & & & 32. \\
\hline $\mathrm{TF} / \mathrm{PF} / \mathrm{div} / \mathrm{mag}$ noz & eq' & & charge) & 0.320 \\
\hline Prop/fuel tankage & “" & "“ & “" & 0.148 \\
\hline Battery re-charge & “ & " & “ & 0.278 \\
\hline Communications & “ & “ & “. & 0.2 \\
\hline Payload & “ & “ & “ & 0.03 \\
\hline Avionics & “ & “ & “ & 0.02 \\
\hline Fuel injector & & & & 0.004 \\
\hline Total (MW) & & & & 400. \\
\hline
\end{tabular}

Should the fusion reactor need to be re-started during the interplanetary transit, the same startup sequence would be followed. The startup reactor power would be used to maintain the refrigeration to the $\mathrm{TF} / \mathrm{PF} /$ divertor/magnetic nozzle coils (to prevent them from "going normal") and propellant/fuel tankage, and to provide for crew payload accommodations, communications, and maintain vehicle avionics. Should the battery bank fail to re-start the reactor, sufficient startup reactor power would be available to re-charge the bank in 35 minutes for another attempt while maintaining power to the other essential systems. Table 12 illustrates the essential power requirements during the re-charge of the re-start battery bank, as well as a summary of the nominal power usage. 


\section{REACTION CONTROL}

Positive control of a vehicle's attitude requires arresting various torques associated with natural perturbations as well as applying torques to achieve vehicle orientations and assist in steering. Most of the significant natural perturbations present in planetary orbit (such as gravity gradient-driven apsidal and nodal drifts, atmospheric drag, magnetic field interactions, etc.) are either significantly diminished or not applicable to interplanetary travel. The torque associated with solar radiation pressure was assessed due to the considerable surface area of the radiator. It was found that even the worst case orientation at Earth solar distance, the radiation torque was negligible.

Main propulsion steering assistance would, however, be needed. The single nozzle configuration would not be able to perform roll control. Pitch and yaw could conceivably be accomplished by selective configuring of the nozzle's magnetic field geometry ${ }^{69}$. This would appear to be the viable way to steer the vehicle using the nozzle, given that the complex coupling between it and the reactor diverter would make gimballing of the nozzle unlikely. However, since reaction control was difficult to assess without a vehicle system control model, and the need for roll control, and the yet to be defined nature of selective configuration of the nozzle's magnetic field geometry, a separate reaction control system (RCS) was added to the design concept.

RCS propulsion technology for today's satellites is transitioning from $\mathrm{N}_{2} \mathrm{H}_{4}$ monopropellants to more advanced technology electric resistojets and arcjets. Since the vehicle concept was significantly more massive than today's spacecraft (requiring more impulse) and since large quantities of electric power and hydrogen would be available, the higher $I_{s p}$ hydrogen fueled arcjet was chosen for the RCS.

Since a vehicle system control model was not available to guide the design of the RCS from a "needs up", a top down approach was pursued to determine how much control authority would exist for a reasonable impulse, power, propellant, and mass allocation. An initial estimate of $500 \mathrm{lb}_{\mathrm{f}}$ per engine, requiring an excessive $50 \mathrm{MW}$ input power per engine, was scaled on the mass and thrust of the current Titan IV/Centaur RCS system. Re-estimation for 8 MW/thruster (i.e. two jets firing per couple, with one fore couple and one aft couple) yielded a thrust level of over $80 \mathrm{lb}_{f}$ per thruster, implying a longer firing time to deliver the same impulse. The RCS thruster parameters were calculated based on the NASA LeRC $30 \mathrm{~kW}$ hydrogen arcjet development program. That program produced thrusters delivering $\mathrm{l}_{\mathrm{sp}}$ 's up to $1,460 \mathrm{lb}_{\mathrm{f}}$ $\mathrm{sec} / \mathrm{lb}_{\mathrm{m}}$ and efficiencies of $30+\%{ }^{70}$. Using these values, mass and mass flow rates per thruster were extrapolated to be $2 \mathrm{mt}$ and $0.0235 \mathrm{~kg} / \mathrm{sec}$ respectively.

The RCS was composed of two units of twelve thrusters each. Each unit housed four thruster clusters 90 degrees apart. Each cluster contained three thrusters, each aimed at right angles to the others. Full six dimensions of freedom control was provided with both units firing simultaneously. One unit was mounted on the aft end of the truss network, forward of the reactor. The other unit was forward of the propellant tanks. It was decided not to place the forward unit at the front of the vehicle since $87 \%$ of the vehicle's fully loaded mass (and $78 \%$ of its dry mass) was aft of the radiators.

An angular velocity of $1.6 \mathrm{deg} / \mathrm{min}$ could be produced by four RCS thrusters firing about the minimum axis (roll) with a torque of $\sim 2,200 \mathrm{~N}-\mathrm{m}$ and a firing time of one minute. For a pitch or yaw maneuver associated with steering, the greater torques $(22,780 \mathrm{~N}$ $\mathrm{m}$ ) due to larger moment arms (mitigated somewhat by the greater moment of inertias about those axes) required only 20 seconds of firing time to produce the same angular rate. In both instances, the angular velocity and the firing times appeared reasonable. The total propellant consumption, assuming five 60 second start/stop corrections per day, every day (up to one year) was almost $21 \mathrm{mt}$. The total mass of the 24 thruster system was $48 \mathrm{mt}$, assuming all power processing was performed by the primary power conversion system.

\section{WEIGHT GROWTH CONTINGENCY}

Weight growth contingency is a margin allocatec to compensate for the inevitable growth in mass experienced by aerospace systems as designs mature and construction takes place. Underestimates become apparent and technical problems are solved by incorporating solutions requiring additional mass. All new lauinch vehicle development programs carry such an allocation, though the percentage allowable varies as a function of component maturity. Experience with the develop nent of eighteen major aerospace vehicles has demons rated that from the point of initial contract proposal through acquisition of first unit, the total average weight growth experienced by military aerospace vehicles has been $25.5 \%{ }^{71}$. For more than a half dcizen major NASA manned and unmanned vehicles and spacecraft, from the point of phase $C / D$ to first velicle flight, most programs have experienced a similar $20 \%$ to $30 \%$ weight growth ${ }^{72}$. Given the immaturity of the overwhelming majority of this technology, past aerospace experience would suggest a prudent minimum value of $30 \%$ weight growth 
allowance be assessed on the total dry mass of the propulsion system $\left(M_{s}\right)$. Thus, a weight growth contingency of $345 \mathrm{mt}$ was carried, representing a major mass property component.

\section{FUTURE AREAS OF ANALYSIS}

Due to time constraints, several potentially superior design solutions were not pursued. Further study is expected to be performed and possibly incorporated into a future upgrade of the design concept. Some potential design alternatives include: inclusion of co-axial helicity injection to reduce the requirement for $\mathrm{N}-\mathrm{NBI}$ and a significant power conversion system; reduced absorption of waste radiation together with a more efficient power conversion system to reduce radiator size and mass; a more rigorous heat transfer analysis; assessment into the feasibility of gray body heat rejection from the $\mathrm{LiH}$ blankets directly to space; replacement of most of the RCS by altering the strength and geometry of the magnetic nozzle's field; and a liquid nitrogen or neon refrigeration cycle for cooling of the TF/PF coils.

\section{CONCLUSIONS}

A conceptual vehicle system design predicated on a small aspect ratio spherical torus nuclear fusion reactor has the potential for enabling relatively fast outer solar system travel. The requirements for a human mission to Saturn were satisfied with a $108 \mathrm{mt}$ payload mass, a 235 day one way trip time, and a IMLEO of $2,941 \mathrm{mt}$. The same concept was found to deliver the same payload to Jupiter requiring a 150 day one way trip time. Both missions presuppose the availability of in situ planetary refueling capability. High orbit space basing was assumed, but not required. A requirementdriven approach provided guidance during the design of the concept, though much of the design decisions were driven as much by the desire to maintain a balance between performance capability and reasonable extrapolations from current technology expectations. A complete vehicle concept was produced with analysis to a sufficient level to make certain assessments on general concept viability and research requirements. Systems analysis, design, and assessment was performed on in-space operations, payload, central truss, nuclear reactor (including divertor and fuel injector), magnetic nozzle, power conversion (turbine, generator, radiator, conditioning), neutral beam injector, refrigeration, tankage, avionics, start-up reactor and battery bank, communications, and reaction control systems. Detailed assessment was performed on reactor operations, including plasma characteristics, power balance, power utilization, and component design. Overall feasibility of nuclear fusion propulsion systems must include assessments of support systems such as heavy lift launch vehicles, space based orbit transfer vehicles, and in situ resource utilization with associated cost of their operations. Critical areas of research upon which the feasibility of this concept rests include the demonstration of ignited, long term steady state, $D^{3} \mathrm{He}$ fueled nuclear fusion reactor operation, the successful incorporation of a divertor able to transfer large quantities of transport power to a propulsion system, the determination whether large downstream propellant infusion adversely impacts fusion reactor operation, the identifying of operations incompatible with spin polarized fuel, and the successful demonstration of a high thrust magnetic nozzle. Once such issues are addressed, a judgement can be rendered as to the practicality of a solar system-class, nuclear fusion-based transportation system of the $21^{\text {st }}$ century.

\section{ACKNOWLEDGMENTS}

The authors wish to thank many who have been very helpful in providing guidance and expertise: to Gerald Hale of Los Alamos National Lab (LANL) in the area of spin polarized $D^{3} \mathrm{He}$ fuel, to Stanley Kaye of Princeton Plasma Physics Lab (PPPL) in the area of fusion confinement time scaling laws, to Joseph Warner of NASA Lewis Research Center (NASA LeRC) in the area of YBCO superconductor material properties and prospects, to Ronald Moses of LANL in the area of Dshape magnet designs and forces, to Lawrence Green of Westinghouse Science and Technology Center and Daniel Driemeyer of Boeing Co. in the area of ITER divertor design drivers, to Peter Turchi and Hani Kamhawi of Ohio State University in the area of magnetic nozzle design and state conditions, to Larry Grisham of PPPL and Masaaki Kuriyama of the Japan Atomic Energy Research Institute in the area of negative ion neutral beam injectors, to Richard Kunath of NASA LeRC in the area of $\mathrm{Ka}$ band space communications, to Robert Cataldo of NASA LeRC in the areas of start-up $\mathrm{NiH}$ batteries and Mars human payload systems; to John Miller of JME, Inc. and Mary Ellen Roth of NASA LeRC in the area of high energy battery capacitors, to John Sankovic of NASA LeRC and R. Joseph Cassady of Primex Technologies Corp. in the area of high power hydrogen arcjets, to Mellissa McGuire of Analex Corp. in the area of reaction control, to Judith Watson of NASA Langley Research Center in the area of Space Station truss network, and to Mohamed Bourham of North Carolina State University and Stanley Milora of Oak Ridge National Lab in the area of fuel injectors. 


\section{REFERENCES}

' Williams, C.H. and Borowski, S.K., "An Assessment of Space Propulsion Fusion Concepts and Desired Operating Parameters for Fast Solar System Travel", AIAA paper 97-3074, July 1997.

${ }^{2}$ Williams, C.H. and Borowski, S.K., "CommerciallyDriven Human Interplanetary Propulsion Systems: Rationale, Concept, Technology, and Performance Requirements", paper \# 58, $13^{\text {th }}$ Symposium on Space Nuclear Power and Propulsion, Albuquerque, NM, January 1996.

${ }^{3}$ Williams, C.H., "An Analytic Approximation to Very High Specific Impulse and Specific Power Interplanetary Space Mission Analysis", NASA TM 107058, NASA Lewis Research Center, Cleveland, $\mathrm{OH}$, September 1995.

${ }^{4}$ Borowski, S.K., "A Comparison of Fusion / Antiproton Propulsion Systems for Interplanetary Travel", AIAA paper 87-1814, July 1987.

${ }^{5}$ Williams, S.N., "An Introduction to the Use of VARITOP", Jet Propulsion Lab, JPL doc. D-11475, January 1994.

${ }^{6}$ George, J., NASA Lewis Research Center, personal communication, June 1997.

${ }^{7}$ Hyde, R.A., "A Laser-Fusion Rocket for Interplanetary Propulsion", $34^{\text {th }}$ International Astronautical Federation, Budapest, Hungary, October 1983.

${ }^{8}$ Isakowitz, S. J., "International Reference Guide to Space Launch Systems", AIAA, 1991.

${ }^{9}$ Borowski, S. K. et al., "Nuclear Thermal Rocket/Vehicle Design Options for Future NASA Missions to the Moon and Mars", NASA TM-107071, September 1995.

${ }^{10}$ Kulcinski, G. L., "Importance of Helium-3 for the Future", Report of the NASA Lunar Energy Enterprise Case Study Task Force, NASA TM-101652, July 1989.

"Hoffman, S. J. and Kaplan, D. I., editors, "ㅂuman Exploration of Mars: The Reference Mission of the NASA Mars Exploration Study Team", NASA Special Publication 6107, July 1997.
${ }^{12}$ Watson. J., "Design Considerations for an Astronaut Monorail system for Large Space Structures and the Structural Characterization of its Positioning Arm", masters thesis (engineering mechanics), Old Dominion University, August 1992.

${ }^{13}$ Borowski, S. K. and Strickler, D. J., "A ProfileAveraged, Steady State Model for Studying Ignitedand Driven-Tokamak Operations", unpublished ORNL paper, 1985.

${ }^{14}$ Hale, Gerald, Los Alamos National Lab, personal communication, February 1998.

${ }^{15}$ Mitarai, O., et al., "Spin Polarization Effect on Ignition Access Condition for D-T and $D^{3} \mathrm{He}$ Tokamak Fusion Reactors", Fusion Technology, July 1992.

${ }^{16}$ Christiansen, J.P., et al., "Global Energy

Confinement H-Mode Database for ITER", Nuclear

Fusion, vol. 32, no. 2, 1992.

${ }^{17}$ Kaye, S.M., et al., "ITER L Mode Confinement Database", Nuclear Fusion, vol. 37, no. 9, 1997.

${ }^{18} \mathrm{McC}$ ool, S.C., et al., "A Mega-Ampere Spherical Tokamak Design for Beta-Limit and Confinement Studies", Transactions of Fusion Technology, vol. 27, April 1995.

${ }^{19}$ Grisharn, L., Princeton Plasma Physics Lab, personal communication, March 1998.

${ }^{20}$ Alava, M.J., et al., "Bootstrap Drive of D- ${ }^{3} \mathrm{He}$ Tokamak Reactors", Proceedings of the $5^{\text {th }}$ Internaticnal Conference on Emerging Nuclear Energy Systems, 1989.

${ }^{21}$ Devote, R.S., et al., "Projections for a Steady State Tokamak Reactor Based on ITER", UCID 21519, December 1989.

${ }^{22}$ Wittenberg, L.J., et al., "Lunar Source of ${ }^{3} \mathrm{He}$ for Commersial Fusion Power", Fusion Technology, Septemb $: 1986$.

${ }^{23}$ Servici, R. F., "New Superconductor Stands Up to Magnetic Fields", Science, May 1995.

${ }^{24}$ Moses: R.W. and Young, W.C., "Analytic Expressions for Magnetic Forces on Sectored Toroidal Coils", Proc. Eng. Probl. Fussion Res., $6^{\text {th }}$, IEEE, 1976. 
${ }^{25}$ Teller, E., editor, Fusion, vol. 1, Academic Press Pub., 1981.

${ }^{26}$ Boyer, R., et al., editors, "Materials Properties Handbook: Titanium Alloys", ASM International Pub, 1994.

${ }^{27}$ Roth, J.R., Introduction to Fusion Energy, Ibis Pub., 1988.

${ }^{28}$ Baker, C.C., et al., "Technology Implications of Advanced Fusion Fuel Cycles", Proc. International Conf. On Plasma Science, IEEE, 1979.

${ }^{29}$ Davis, J.R., et al., editors, "Metals Handbook", vol. 2, ASM International Pub, 1990.

${ }^{30}$ LaMarsh, J.R., Introduction to Nuclear Engineering, Addison-Wesley Pub., 1977.

${ }^{31}$ Frattolillo, A., et al., "Development of a Two-Stage Pneumatic Repeating Pellet Injector for the Refueling of Long-Pulse Magnetic Confinement Fusion Devices", Fusion Technology, December 1997.

${ }^{32}$ Combs, S.K., et al., "Pellet Injector Research and Development at ORNL,", Fusion Technology, 1994.

${ }^{33}$ Kincaid, R.W. and Bourham, M.A., "Electrothermal Plasma Gun as a Pellet Injector", Fusion Technology, 1994.

${ }^{34}$ Bourham, M.A., North Caroline State University, personal communication, June 1998.

${ }^{35}$ Milora, S.L., Oak Ridge National Lab, personal communication, June 1998.

${ }^{36}$ Carpenter S.A. and Deveny, M.E., "Mirror Fusion Propulsion System (MFPS): An Option for the Space Exploration Initiative (SEI)", Space Technology, vol. 14, No. 1, Elsevier Science Ltd. Pub., 1994.

${ }^{37}$ Post, D. et al., "The ITER Power and Particle Control System", Fusion Technology, December 1996.

${ }^{38}$ Araki, M., et al., "Analytical Study of Electromagnetic Forces Induced By Eddy Current in ITER Divertor", Fusion Technology, December 1996.
${ }^{39}$ Sevier, D.L. et al., "Implications of Steady State Operation on Divertor Design", Fusion Technology, December 1996.

${ }^{40}$ Green, L., Westinghouse Science and Technology Center, personal communication, May 1998.

${ }^{41}$ Cikanek, H.A., "Lewis Research Center Revised Submissions to the Advanced Technology Lead Center for the Fiscal Year 2000 Budget Call", NASA official letter, June 1998.

${ }^{42}$ Gerwin, R. A., et al., "Characterization of Plasma Flow Through Magnetic Nozzles", Astronautics Lab (AFSC) AL-TR-89-092, final report, February 1990.

${ }^{43}$ Kamhawi, H., Ohio State University, unpublished notes, April 1997.

${ }^{44}$ Turchi, P.J., Ohio State University, unpublished notes, April 1997.

${ }^{45}$ Turchi, P.J., Ohio State University, email, June 1998.

${ }^{46}$ Borowski, S.K. and Dudzinski, L.A., “ '2001: A Space Odyssey' Revisited - The Feasibility of 24 Hour Commuter Flights to the Moon Using NTR Propulsion with LUNOX Afterburners", AIAA paper 97-2956, July 1997.

${ }^{47}$ Sutton, G.P. and Ross D.M., Rocket Propulsion Elements, John Wiley \& Sons Pub., 1976.

${ }^{48}$ Keller, C. and Schmidt, D., "Industrial Closed Cycle Gas Turbines for Conventional and Nuclear Fuel", Gas Turbine Products and Turbine Products Show, Houston, Texas, March 5-9, 1967.

${ }^{49}$ Bammert, K., "'A General Review of Closed Cycle Gas Turbines Using Fossile, Nuclear, and Solar Energy", Verlag K. Thiemig, 1975.

${ }^{50}$ Bohm, E., "Gas Turbines for Nuclear Power Plants", off-print for Euratom Review, February 1968.

${ }^{51}$ Juhasz, A.J., et al., "Closed Brayton Cycle Power System with a High Temperature Pellet Bed Reactor Heat Source for Nuclear-Electric Propulsion Applications", NASA TM-105933, October 1992. 
\$2 Kuriyama, M., et al., "High Energy Negative-Ion Based Neutral Beam Injection System for JT-60U", Fusion Engineering and Design, vol. 26, 1995.

${ }^{53}$ Grisham, L., Princeton Plasma Physics Lab, email, May 1998.

${ }^{54}$ Ohara, Y., "Progress of Neutral Beam R\&D for Plasma Heating and Current Drive at JAERI ", Fusion Engineering and Design, vol. 26, 1995.

s5 "Nuclear Reactor/Propulsion System Interaction", General Dynamics Space Systems Division, final report, November 1993.

56 "Space Shuttle System Summary", Rockwell International Space Systems Group, May 1980.

57 "Saturn V News Reference", NASA Marshall Space Flight Center, et al., December 1968.

${ }^{58}$ Kudlac, M., NASA Lewis Research Center, personal communication, June 1998.

\$9 "Nuclear Reactor/Propulsion System Interaction", General Dynamics Space Systems Division, final Report, November 1993.

60 "Advanced Communication Technology Satellite", NASA Fact Sheet, ACTS Pub \#301

${ }^{61}$ Cook, D. and Bauer, B., "Advanced Communications Technology Satellite Program Overview", April 1993.

${ }^{62}$ Kunath, R., NASA Lewis Research Center, personal communication, June 1998.

63 "Space Shuttle System Summary", Rockwell International, May 1980

${ }^{64}$ Borowski, S.K., Peng, Y-K. M., and Kammash, T., "Radio-Frequency-Assisted Current Startup in the Fusion Engineering Device," Fusion Technology, Vol. 6, July 1984.

${ }^{65}$ Borowski, S. K., "Inductive Current Startup in Large Tokamaks With Expanding Minor Radius and RF Assist," Oak Ridge National Laboratory Report FEDC$83 / 8$, February 1984.
${ }^{66}$ Cataldo, R.L., "Test Results of a Ten Cell Bipolar Nickel-Hydrogen Battery", NASA TM-83384, August 1983.

${ }^{67}$ Cataldo, R.L., NASA Lewis Research Center, personal communication, June 1998.

68 "Fastcharged Traction and Starter Supercapacitor Batteries", marketing brochure, ESMA Co., Moscow, Russia, 1998.

${ }^{69}$ Turchi, P.J., Ohio State University, personal communication, June 1998.

${ }^{70}$ Sankovic, J.M., et al., "Hydrogen Arcjet Technology", NASA TM-105340, October 1991.

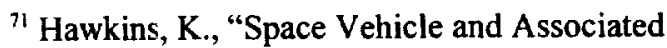
Subsystem Weight Growth", SAWE paper 1816, May 1988.

${ }^{72}$ Anonymous presentation, "MSFC Programs Weight Growth History", April 1979. 



\begin{tabular}{|c|c|c|c|c|}
\hline \multicolumn{3}{|c|}{ REPORT DOCUMENTATION PAGE } & \multicolumn{2}{|r|}{$\begin{array}{l}\text { Form Approved } \\
\text { OMB No. } 0704-0188\end{array}$} \\
\hline \multicolumn{5}{|c|}{ 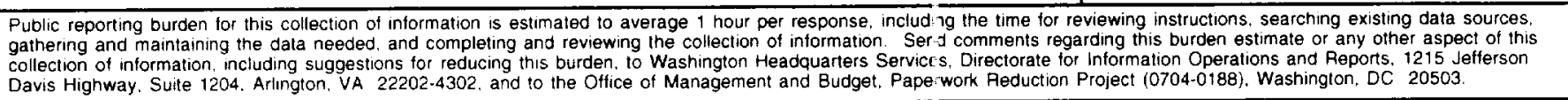 } \\
\hline 1. AGENCY USE ONLY (Leave blank) & $\begin{array}{l}\text { 2. REPORT DATE } \\
\text { December } 1998\end{array}$ & \multicolumn{3}{|c|}{$\begin{array}{l}\text { 3. REPORT TYPE AND DATES COVERED } \\
\text { Technical Memorandum }\end{array}$} \\
\hline \multicolumn{3}{|c|}{$\begin{array}{l}\text { 4. TITLE AND SUBTITLE } \\
\text { A Spherical Torus Nuclear Fusion Reactor Space Propulsion Vehicle Concept } \\
\text { for Fast Interplanetary Travel }\end{array}$} & \multicolumn{2}{|c|}{ 5. FUNDING NUMBERS } \\
\hline \multicolumn{3}{|c|}{$\begin{array}{l}\text { Craig H. Williams. Stanley K. Borowski, Leonard A. Dudzinski, } \\
\text { and Albert J. Juhasz }\end{array}$} & \multicolumn{2}{|c|}{ WU-242-74-74-00 } \\
\hline \multicolumn{3}{|c|}{$\begin{array}{l}\text { 7. PERFORMING ORGANIZATION NAME(S) AND ADDRESS(ES) } \\
\text { National Aeronautics and Space Administration } \\
\text { Lewis Research Center } \\
\text { Cleveland. Ohio } 44135-3191\end{array}$} & \multicolumn{2}{|c|}{ E-11442 } \\
\hline \multicolumn{3}{|c|}{ 9. SPONSORING/MONITORING AGENCY NAME(S) AND ADDRESS(ES) } & \multicolumn{2}{|c|}{$\begin{array}{l}\text { 10. SPONSORING/MONITORING } \\
\text { AGENCY REPORT NUMBER } \\
\text { NASA TM-1998-208831 } \\
\text { AIAA-98-3591 }\end{array}$} \\
\hline \multicolumn{5}{|c|}{$\begin{array}{l}\text { 11. SUPPLEMENTARY NOTES } \\
\text { Prepared for the 34th Joint Propulsion Conference and Exhibit cosponsored by the AIAA. ASME. SAE. and ASEE, } \\
\text { Cleveland. Ohio. July 13-15. 1998. Craig H. Williams, Stanley K. Bolowski, Leonard A. Dudzinski. and Albert J. Juhasz, } \\
\text { NASA Lewis Research Center. Responsible person, Craig H. Williams, organization code } 6510 . \text { (216) 433-7063. }\end{array}$} \\
\hline \multirow{2}{*}{\multicolumn{3}{|c|}{$\begin{array}{l}\text { 12a. OISTRIBUTION/AVAILABILITY STATEMENT } \\
\text { Unclassified-Unlimited } \\
\text { Subject Categories: } 20,15, \text { and } 16 \quad \text { Distribution: Nenstandard } \\
\text { This publication is available from the NASA Center for AeroSpace Information. (c01) 621-0390. }\end{array}$}} & \multirow{2}{*}{\multicolumn{2}{|c|}{ 12b. DISTRIBUTION CODE }} \\
\hline & & & & \\
\hline \multicolumn{5}{|c|}{$\begin{array}{l}\text { A conceptual vehicle design enabling fast outer solar system travel was produced predicated on a small aspect ratio } \\
\text { spherical torus nuclear fusion reactor. Initial requirements were for a human mission to Saturn with a }>5 \% \text { payload mass } \\
\text { fraction and a one way trip time of less than one year. Analysis revealed that the vehicle could deliver a } 108 \mathrm{mt} \text { crew } \\
\text { habitat payload to Saturn rendezvous in } 235 \text { days, with an initial mass in low Earth orbit of } 2.941 \text { mt. Engineering } \\
\text { conceptual design, analysis, and assessment was performed on all major systems including payload. central truss, nuclear } \\
\text { reactor (including divertor and fuel injector), power conversion (incluting turbine, compressor, alternator, radiator, } \\
\text { recuperator, and conditioning), magnetic nozzle, neutral beam injecto:, tankage, start/re-start reactor and battery, refrig- } \\
\text { eration, communications, reaction control, and in-space operations. Detailed assessment was done on reactor operations, } \\
\text { including plasma characteristics, power balance, power utilization, an: component design. }\end{array}$} \\
\hline \multirow{2}{*}{\multicolumn{3}{|c|}{$\begin{array}{l}\text { 14. SUBJECT TEAMS } \\
\text { Nuclear: Fusion: Interplanetary; High thrust; Nuclear propulsion: Satırn; Jupiter; } \\
\text { Spacecraft }\end{array}$}} & & $\begin{array}{l}\text { 15. NUMBER OF PAGES } \\
38\end{array}$ \\
\hline & & & & $\begin{array}{r}\text { 16. PAICE CODE } \\
\text { A03 }\end{array}$ \\
\hline $\begin{array}{l}\text { 17. SECURITY CLASSIFICATION } \\
\text { OF REPORT }\end{array}$ & $\begin{array}{l}\text { 18. SECURITY CLASSIFICATION } \\
\text { OF THIS PAGE }\end{array}$ & $\begin{array}{l}\text { 19. SECUAITY CLASSIFIC } \\
\text { OF AESTRACT }\end{array}$ & ATION & 20. LIMITATION OF ABSTRACT \\
\hline Unclassified & Unclassified & Unclassified & & \\
\hline
\end{tabular}

